\title{
A walk in the maze: Variation in Late Jurassic tridactyl dinosaur tracks from the Swiss Jura Mountains (NW Switzerland)
}

\author{
Diego Castanera ${ }^{\text {Corresp., }}{ }^{1}$, Matteo Belvedere ${ }^{2}$, Daniel Marty ${ }^{2}$, Géraldine Paratte ${ }^{2}$, Marielle Lapaire-Cattin ${ }^{2}$, \\ Christel Lovis $^{2}$, Christian A Meyer ${ }^{3}$ \\ ${ }^{1}$ GeoBioCenter, Ludwig-Maximilians-Universität, Bayerische Staatssammlung für Paläontologie und Geologie, Munich, Germany \\ 2 Section d'archéologie et paléontologie, Paléontologie A16, Office de la culture,, Porrentruy, Switzerland \\ 3 Department of Environmental Sciences, University of Basel, Basel, Switzerland \\ Corresponding Author: Diego Castanera \\ Email address: dcastanera@unizar.es
}

Background. Minute to medium-sized (FL less than $30 \mathrm{~cm}$ ) tridactyl dinosaur tracks are the most abundant in the Late Jurassic tracksites of Highway A16 (Reuchenette Formation, Kimmeridgian) in the Jura Mountains (NW Switzerland). During excavations, two morphotypes, one gracile and one robust, were identified in the field. Furthermore, two large-sized theropod ichnospecies (Megalosauripus transjuranicus and Jurabrontes curtedulensis) and an ornithopod-like morphotype (Morphotype II) have recently been described at these sites. Methods. The quality of morphological preservation (preservation grade), the depth of the footprint, the shape variation and the footprint proportions (FL/FW ratio and mesaxony) along the trackways have been analysed using 3D models and false-colour depth maps in order to determine the exact number of small to medium-sized morphotypes present in the tracksites. Results. The study of footprints ( $\mathrm{n}=$ 93) recovered during the excavations has made it possible to identify and characterize the two morphotypes distinguished in the field. The gracile morphotype is mainly characterized by a high footprint length/width ratio, high mesaxony, low divarication angles and clear, sharp claw marks and phalangeal pads (2-3-4). By contrast, the robust morphotype is characterized by a lower footprint length/width ratio, weaker mesaxony, slightly higher divarication angles and clear, sharp claw marks (when preserved), whereas the phalangeal pads are not clearly preserved although they might be present.

Discussion. The analysis does not allow the two morphotypes to be associated within the same morphological continuum. Thus, they cannot be extramorphological variations of similar tracks produced by a single trackmaker. Comparison of the two morphotypes with the larger morphotypes described in the formation (Megalosauripus transjuranicus, Jurabrontes curtedulensis and Morphotype II) and the spatio-temporal relationships of the trackways suggest that the smaller morphotypes cannot reliably be considered as small 
individuals of any of the larger morphotypes. The morphometric data of some specimens of the robust morphotype (even lower values for the length/width ratio and mesaxony) suggest that more than one ichnotaxon might be represented within the robust morphotype. The features of the gracile morphotype (cf. Kalohipus isp.) are typical of "grallatorid" ichnotaxa with low mesaxony whereas those of the robust morphotype (cf. Therangospodus isp. and Therangospodus? isp.) are reminiscent of Therangospodus pandemicus. This work sheds new light on combining an analysis of variations in footprint morphology through 3D models and false-colour depth maps, with the study of possible ontogenetic variations and the identification of small-sized tridactyl ichnotaxa for the description of new dinosaur tracks. 
1 A walk in the maze: Variation in Late Jurassic tridactyl dinosaur tracks from the Swiss Jura

2 Mountains (NW Switzerland)

3

4 Diego Castanera ${ }^{1}$, Matteo Belvedere ${ }^{2}$, Daniel Marty ${ }^{2}$, Géraldine Paratte ${ }^{2}$, Marielle Lapaire-

5 Cattin $^{2}$, Christel Lovis ${ }^{2}$, Christian A. Meyer ${ }^{3}$

6 Bayerische Staatssammlung für Paläontologie und Geologie and GeoBioCenter, Ludwig-

7 Maximilians-Universität Munich, Munich, Germany

8 2Office de la culture, Section d'archéologie et paléontologie, Paléontologie A16, Porrentruy,

9 Switzerland.

$10{ }^{3}$ Department of Environmental Sciences, University of Basel, Basel, Switzerland

11

12

Corresponding author: Diego Castanera

Email address: dcastanera@hotmail.es; d.castanera@lrz.uni-muenchen.de 


\section{ABSTRACT}

Background. Minute to medium-sized (FL less than $30 \mathrm{~cm}$ ) tridactyl dinosaur tracks are the most abundant in the Late Jurassic tracksites of Highway A16 (Reuchenette Formation, Kimmeridgian) in the Jura Mountains (NW Switzerland). During excavations, two morphotypes, one gracile and one robust, were identified in the field. Furthermore, two large-sized theropod ichnospecies (Megalosauripus transjuranicus and Jurabrontes curtedulensis) and an ornithopodlike morphotype (Morphotype II) have recently been described at these sites.

Methods. The quality of morphological preservation (preservation grade), the depth of the footprint, the shape variation and the footprint proportions (FL/FW ratio and mesaxony) along the trackways have been analysed using 3D models and false-colour depth maps in order to determine the exact number of small to medium-sized morphotypes present in the tracksites.

Results. The study of footprints $(n=93)$ recovered during the excavations has made it possible to identify and characterize the two morphotypes distinguished in the field. The gracile morphotype is mainly characterized by a high footprint length/width ratio, high mesaxony, low divarication angles and clear, sharp claw marks and phalangeal pads (2-3-4). By contrast, the robust morphotype is characterized by a lower footprint length/width ratio, weaker mesaxony, slightly higher divarication angles and clear, sharp claw marks (when preserved), whereas the phalangeal pads are not clearly preserved although they might be present.

Discussion. The analysis does not allow the two morphotypes to be associated within the same morphological continuum. Thus, they cannot be extramorphological variations of similar tracks produced by a single trackmaker. Comparison of the two morphotypes with the larger morphotypes described in the formation (Megalosauripus transjuranicus, Jurabrontes curtedulensis and Morphotype II) and the spatio-temporal relationships of the trackways suggest that the smaller morphotypes cannot reliably be considered as small individuals of any of the larger morphotypes. The morphometric data of some specimens of the robust morphotype (even lower values for the length/width ratio and mesaxony) suggest that more than one ichnotaxon might be represented within the robust morphotype. The features of the gracile morphotype (cf. Kalohipus isp.) are typical of "grallatorid" ichnotaxa with low mesaxony whereas those of the robust morphotype (cf. Therangospodus isp. and Therangospodus? isp.) are reminiscent of Therangospodus pandemicus. This work sheds new light on combining an analysis of variations in footprint morphology through 3D models and false-colour depth maps, with the study of possible ontogenetic variations and the identification of small-sized tridactyl ichnotaxa for the description of new dinosaur tracks. 
65

66

67

68

69

70

71

72

73

74

75

76

77

78

79

80

81

82

83

84

85

86

87

88

89

90

91

92

93

94

95

96

97

98

99

100

101

\section{INTRODUCTION}

Since the first sauropod tracks were reported in the Lommiswil quarry (Late Kimmeridgian, Canton Solothurn) in the Swiss Jura Mountains (Meyer, 1990), dinosaur track discoveries have increased considerably, and to date more than 25 tracksites have been documented in the cantons of Jura, Bern, Neuchâtel and Solothurn. Most of these tracksites occur in the Reuchenette Formation (Kimmeridgian), and some of them in the Twannbach Formation (Tithonian) (Meyer \& Thüring, 2003; Marty, 2008; Marty \& Meyer, 2012; Marty et al., 2010; 2013). Between 2002 and 2011, six large tracksites were systematically excavated and documented by the Palaeontology A16 (Marty and Billon-Bruyat, 2009) prior to the construction of Highway A16 in the Canton Jura (NW Switzerland). These tracksites covered together a surface area of 18,500 $\mathrm{m}^{2}$, and a total of 59 ichnoassemblages. Out of 14,000 individual tracks, 254 trackways were attributed to sauropods and 411 to bipedal tridactyl dinosaurs. Therefore, the Jura carbonate platform has today become a key area for Late Jurassic dinosaur palaeoichnology (Marty, 2008; Marty \& Meyer, 2012) as it represents one of the areas with the highest number of Late Jurassic dinosaur tracks in the world.

Recent papers have described giant theropod tracks (Jurabrontes curtedulensis, Marty et al., 2017) and large theropod tracks (Megalosauripus transjuranicus, Razzolini et al., 2017) from the Swiss Jura Mountains, but most of the tridactyl tracks by far are the still largely undescribed minute, small and medium-sized tracks (footprint length $<30 \mathrm{~cm}$ ). Marty (2008) described minute and small tridactyl tracks from the Chevenez-Combe Ronde tracksite (Canton Jura, NW Switzerland) and tentatively attributed some of these to Carmelopodus. Since then, however, many other tracksites and ichnoassemblages with minute to medium-sized tridactyl tracks have been discovered, including some very well-preserved tracks of different morphotypes and some very long trackways (up to $100 \mathrm{~m}$ ).

In Europe, apart from the Swiss and French (Mazin, Hantzpergue \& Pouech, 2016) Jura Mountains, the main Late Jurassic deposits that have yielded minute to medium-sized tridactyl dinosaur tracks are located in the Lusitanian Basin in Portugal (Antunes \& Mateus, 2003; Santos, 2008), the Asturian Basin in Spain (Lockley et al., 2008; Piñuela, 2015), the Aquitanian Basin in France (Lange-Badré et al., 1996; Mazin et al., 1997; Moreau et al., 2017), the Lower Saxony Basin in NW Germany (Kaever \& Lapparent, 1974; Diedrich, 2011; Lallensack et al., 2015), and several units in the Holy Cross Mountains in Poland (Gierliński, Niedźwiedzki \& Nowacki, 2009). The units that date to around the Jurassic-Cretaceous boundary (Tithonian-Berriasian) in the Iberian Range in Spain (Santisteban et al., 2003; Castanera et al., 2013a; Alcalá et al., 2014; Campos-Soto et al., 2017) should also be mentioned. It is noteworthy that, while there is a high number of small to medium-sized tridactyl tracks (assigned to both theropods and ornithopods) described, only few ichnotaxa have been formally erected so far. Besides the tracks from the Chevenez-Combe Ronde tracksite tentatively assigned to Carmelopodus by Marty (2008), the 
102

103

104

105

106

107

108

109

110

111

112

113

114

115

116

117

118

119

120

121

122

123

124

125

126

127

128

129

130

131

132

133

134

135

136

137

138

main small to medium-sized tridactyl tracks identified have been from Spain (Grallator and Anomoepus, from several sites in Asturias, Lockley et al., 2008; Piñuela, 2015; Castanera, Piñuela \& García-Ramos, 2016), France (Carmelopodus, Loulle tracksite, Mazin, Hantzpergue \& Pouech, 2016), Poland (Wildeichnus, cf. Jialingpus and Dineichnus, different units in the Holy Cross Mountains, Gierliński, Niedźwiedzki \& Nowacki, 2009), Germany (Grallator, Bergkirchen tracksite, Diedrich, 2011) and Portugal (Dineichnus and ?Therangospodus, Lockley et al., 1998a; Lockley, Meyer \& Moratalla, 2000). Other significant Late Jurassic areas with minute to medium-sized tridactyl dinosaur tracks are found in the USA (Foster \& Lockley, 2006), Morocco (Belvedere, Mietto \& Ishigaki, 2010), China (Xing, Harris \& Gierliński, 2011; Xing et al., 2016), Yemen (Schulp \& Al-Wosabi, 2012) and Turkmenistan (Lockley, Meyer \& Santos, 2000; Fanti et al., 2013).

Several recent papers have examined the variability in track morphology along trackways (Razzolini et al., 2014, 2017; Lallensack, van Heteren, \& Wings, 2016), showing how pronounced changes can occur along a single trackway. Thus, sometimes it can be very difficult to determine the exact number of ichnotaxa and clearly distinguish between them, especially when the tracks are morphologically similar. This should be borne in mind particularly when studying the material from Switzerland, where large theropod tracks have shown notable variations in shape along the same trackway (Razzolini et al., 2017). In the case of the minute to medium-sized tridactyl tracks, two different morphotypes were identified at first glance during the documentation of the tracksites, one gracile and one more robust type. The aim of this paper is to describe the small to medium-sized tridactyl tracks collected in the Jura Mountains (NW Switzerland). Special emphasis is put on the analysis of track morphology through 3D models and possible variations in footprint shape along trackways in order to find out if the different morphotypes are a consequence of preservation. In addition, other factors such as possible ontogenetic variations in the larger ichnospecies described in the formation are also taken into account. Finally, we discuss the ichnotaxonomy of the tracks.

\section{GEOGRAPHICAL AND GEOLOGICAL SETTING}

The studied material comes from six different tracksites from Highway A16 and nearby areas (Fig. 1A): (1) Courtedoux-Bois de Sylleux (CTD-BSY), (2) Courtedoux-Tchâfouè (CTDTCH), (3) Courtedoux-Béchat Bovais (CTD-BEB), (4) Courtedoux - Sur Combe Ronde (CTD-SCR), (5) Chevenez-Combe Ronde (CHE-CRO); and (6) Chevenez-La Combe (CHE$\mathrm{CHV}$ ). For the sake of simplicity BSY, TCH, BEB, SCR, CRO and CHV are used in the publication.

All the tracksites are located in the Ajoie district about 6-8 $\mathrm{km}$ to the west of Porrentruy (Canton Jura, NW Switzerland) on the track of Swiss federal highway A16 except the Chevenez-La Combe tracksite, which is located in a quarry near the village of Chevenez. The first five 
139

140

141

142

143

144

145

146

147

148

149

150

151

152

153

154

155

156

157

158

159

160

161

162

163

164

165

166

167

168

169

170

171

172

173

174

175

tracksites were systematically excavated level-by-level by the Palaeontology A16 (PALA16) from 2002 until 2011 (Marty et al., 2003; Marty et al., 2004; Marty et al., 2007; Marty, 2008; Marty and Billon-Bruyat, 2009).

Geologically, the study area belongs to the Tabular Jura Mountains and is located at the eastern end of the Rhine-Bresse transfer zone between the Folded Jura Mountains (South and East) and the Upper Rhine Graben and Vosges Mountains (North). The Upper Jurassic strata of the Swiss Jura Mountains are made up of shallow-marine carbonates deposited on the large and structurally complex Jura carbonate platform, which was located at the northern margin of the Tethys at a palaeolatitude of approximately $30^{\circ} \mathrm{N}$ (Thierry, 2000; Thierry et al., 2000; Stampfli \& Borel, 2002).

The tracksites occur within the Reuchenette Formation (Kimmeridgian), dated by ammonites of the Cymodoce to Mutabilis (Boreal), and Divisum to Acanthicum (Tethyan) biozones (Comment et al., 2015). Accordingly, the age of the track-bearing levels is late Early to early Late Kimmeridgian (Gygi, 2000; Jank et al. 2006). This age is also confirmed by the presence of ostracods (Schudack et al., 2013). More information on the sedimentology and palaeoenvironment of the Highway A16 tracksites can be found in Jank et al. (2006), Marty (2008), Razzolini et al. (2017) and Marty et al. (2017).

Stratigraphically, the tracksites include three different track-bearing laminite intervals, separated by shallow marine limestones (Marty, 2008; Waite et al., 2008; Comment, Ayer \& Becker, 2011; Comment et al., 2015). The three main track-bearing laminite intervals are referred to as the lower, intermediate and upper levels, respectively levels 500-550, 1000-1100, and 1500-1650 (Fig. 1B). Only tracks from the lower and intermediate track levels are included in the present study (Fig. 1B), and the studied tracks come from a total of 11 different ichnoassemblages (stratigraphic track levels). These are as follows: BEB500, CRO500, BSY1020, BSY1040, BSY1050, TCH1055, SCR1055, TCH1060, TCH1065, TCH1069 and CHV1000-1100 (precise level cannot be indicated).

\section{MATERIAL AND METHODS}

We analysed a total of 93 individual tracks (Table S1) that are housed in the track collection of PAL A16 (Canton Jura), either as original specimens or as replicas. This collection will be transferred to JURASSICA Muséum (Porrentruy, Canton Jura) in 2019. All the tracks are from the aforementioned tracksites, the largest samples coming from BEB500 (39 footprints), TCH1065 (15) and CRO500 (20). Each analysed track has two acronyms (Table S1): one represents the number of the slab within the collection, e.g.: TCH006-1100 denotes Tchâfouè tracksite, year 2006 (the year of discovery), slab 1100 (an "r" in front of the specimen number, means replica). Some high-resolution laser scans were made in the field and those tracks are here referred to as "Laser-Scan". A second acronym represents the level and number of the trackway 
176

177

178

179

180

181

182

183

184

185

186

187

188

189

190

191

192

193

194

195

196

197

198

199

200

201

202

203

204

205

206

207

208

209

210

211

212

213

214

and track, e.g.: TCH1055-T2-L1 denotes Tchâfouè tracksite, level 1055, trackway 2, track 1, left pes. The second acronym is used throughout the manuscript. As the track-bearing layers were excavated level-by-level there are no doubts about the preservation mode of the tracks. Thus, all the tracks were preserved as true tracks (concave epireliefs) and were produced on the tracking surface, with the only exception of TCH1060-E58, which was preserved as a natural cast (convex hyporelief).

Analysis of track morphology was performed independently for each track; however, some tracks belong to trackways, therefore their variation in morphology along a single trackway was also considered in order to avoid over-identification of morphotypes. These trackways are: BEB500-T16 (3), BEB500-T17 (4), BEB500-T58 (6), BEB500-T73 (4), BEB500-T75 (2), BEB500-T78 (2), BEB-500-T82 (2), BEB-500-T93 (2), BEB500-T120 (4), CRO500-T10 (14), CRO500-T30BIS (5), TCH1055-T2 (2), TCH1065-T15 (2), TCH1065-T25 (2) and TCH1069-T2 (2). We analysed each individual track and made an evaluation of the quality of preservation according to the scale of Belvedere and Farlow (2016) (Table S1). As stated by these authors, "quantitative shape analyses need to be based on data of high quality, and comparisons are best made between tracks comparable in quality of preservation". Accordingly, only the tracks with a preservation grade equal to or higher than 2 were considered for measurement and analysed in this paper; field measurements exist for all the other tracks and are stored in the PALA16 database. The descriptions are based on identification of two different morphotypes, one gracile and one robust, during the documentation in the field. Thus, the footprint length (FL), footprint width (FW), length and width of digits II (LII, WII), III (LIII, WIII) and IV (LIV, WIV), divarication angles (II-III; III-IV) were measured (see Castanera, Piñuela \& García-Ramos, 2016, fig. 2). Subsequently, the FL/FW ratio and the mesaxony were calculated. The latter was calculated on the basis of the anterior triangle length-width ratio (AT) following Lockley (2009). All these measurements were taken from perpendicular pictures with the software Image J. The tracks were classified according to different size classes (Marty, 2008) on the basis of pes length (FL) as: 1) minute, $\mathrm{FL}<10 \mathrm{~cm}$; 2) small, $10 \mathrm{~cm}<\mathrm{FL}<20 \mathrm{~cm}$; 3) medium, $20 \mathrm{~cm}<\mathrm{FL}<30 \mathrm{~cm}$; and 4) large, FL $>30 \mathrm{~cm}$. The morphometric data of the studied tracks were compared in a bivariate plot (length/width ratio vs. mesaxony) with larger tracks (Megalosauripus transjuranicus, Jurabrontes curtedulensis and Morphotype II) described in the Reuchenette Formation (Razzolini et al., 2017; Marty et al., 2017). In addition, they were also compared with other theropod ichnotaxa using data from Castanera, Piñuela \& García-Ramos (2016) which were mainly compiled after Lockley (2009) and Xing et al. (2014). Data were analysed with the software PAST v.2.14 (Hammer, Harper \& Ryan, 2001).

3D-photogrammetric models were generated from pictures taken with a Canon EOS 70D camera equipped with a Canon 10-18mm STL lens using Agisoft Photoscan (v. 1.3.2, www.agisoft.com) following the procedures of Mallison \& Wings (2014) and Matthews, Noble \& Breithaupt (2016). Within the BEB500 sample, 3D data of 10 footprints were obtained by high-resolution laser-scanning carried out in the field in 2011 by Pöyry AG with a Faro hand-scanner. Most of 
215 these 10 footprints were destroyed during the construction of Highway A16. The scaled meshes 216 were exported as Stanford PLY files (.ply) and then processed in CloudCompare (v.2.7.0, 217 www.cloudcompare.com) in order to obtain accurate false-colour depth maps. All 218 photogrammetric meshes used in this study are available for download here: 219 https://doi.org/10.6084/m9.figshare.5662306 (ca. $2.5 \mathrm{~Gb}$ ). In addition, we analysed the 220 maximum depth of all the tracks, in order to ascertain whether there is a relationship between 221 depth, preservation grade and the morphotype. The maximum depth was estimated using the

222 false-colour map derived from the 3D-model in those tracks with a preservation grade generally 223 higher than 0.5 .

224

\section{DESCRIPTION OF THE TRACK MORPHOTYPES AND MORPHOLOGICAL} VARIATIONS ALONG THE TRACKWAYS:

Gracile morphotype:

This morphotype was identified in all six tracksites. The footprints are small to medium-sized $(15-21.2 \mathrm{~cm})$ tridactyl tracks (Fig. 2), clearly longer than wide $(\mathrm{FL} / \mathrm{FW}$ ratio $=1.50-1.90)($ Table 1). The digits are slender with an acuminate end and clear claw marks preserved in the three digits in the majority of the tracks. Digit III is clearly longer and slightly wider than digits II and IV. Digits II and IV are similar in length and width. The mesaxony is variable but medium to high (AT $=0.53-0.98)$, with a mean value of 0.77 , although it is higher in most of the specimens (more than 0.8 in half of the sample). The divarication angles are relatively low, II-III generally being slightly higher (mean $25^{\circ}$ ) than III-IV (mean $22^{\circ}$ ). The hypices are quite symmetrical. The "heel" morphology is variable; some specimens have an oval to round heel pad connected with digit IV (BEB500-T16-R3, TCH1055-E53, TCH1055-T2-R1, TCH1069-T1-R2; see Fig. 2), whereas in others it is not clearly preserved even when the preservation grade is high (e.g.: BSY1020-E2). Most of the specimens preserve a clear small medial notch located behind digit II, which with the rounded heel marks gives them an asymmetric shape. In some of the footprints well-defined digital pads can be discerned. The tracks with the best quality of preservation suggest a phalangeal formula of 2-3-4 (including the metatarsophalangeal pad IV).

\section{Robust morphotype:}

This morphotype has mainly been identified on the track levels BEB500 and TCH1065. The footprints are small or medium-sized $(17-21.8 \mathrm{~cm}$ ) tridactyl tracks (Fig. 3), slightly longer than wide $(\mathrm{FL} / \mathrm{FW}$ ratio $=1.13-1.46)$, (Table 1$)$. The digits are relatively robust with an acuminate end and clear claw marks preserved in some of the tracks (e.g.: BEB500-T120-R5, TCH1065T15-R1, TCH1065-T21-R1). Digit III is clearly longer and slightly wider than digits II and IV. Digits II and IV are similar in length and width. The mesaxony is variable but low-medium (AT $=0.38-0.61$ ), with a mean value of 0.49 . The divarication angles are low, II-III (mean $26^{\circ}$ ) and III-IV (mean $27^{\circ}$ ) being quite similar. The hypices are quite symmetrical. The "heel" morphology is variable, ranging from subrounded to subtriangular. Only TCH1065-T21-R1 
252 preserves a clear small medial notch located behind digit II, thus being slightly asymmetrical, 253 whereas the other specimens are more symmetrical. Well-defined digital pads cannot be 254 discerned in most of the footprints, although TCH1065-T21-R1 shows digital pads suggesting a 255 possible phalangeal pad formula of 2-3-?4.

256 Table 2 and Figs. 4 and 5 show the variations in preservation grade and the maximum depth along the analysed trackways (see Table S1 to see the data of each specific track). 11 trackways

258

259

260

261

262

263

264

265

266

267

268

269

270

271

272

273

274

275

276

277

278

279

280

281

282

283

284

285

286

287

288

289 belong to the gracile morphotype and 4 trackways belong to the robust morphotype (see Figs. $\mathrm{S} 1-\mathrm{S} 3$ for the location of the trackways in the tracksite). The preservation grade varies from low (0-0.5 in the scale) to high ( 2 or more in the scale) for both morphotypes. The maximum depth variation is considerably low, as the maximum variation it is around $5 \mathrm{~mm}$ in both morphotypes (CRO500-T30BIS and BEB500-T120),

\section{DISCUSSION:}

1) True ichnodiversity or variation due to substrate-foot interaction?

The final shape of a footprint is determined by a combination of factors related to the anatomy of the trackmaker's autopodium, the kinematics and the substrate (Marty et al., 2009; Falkhingham, 2014); another important factor is the level in which the tracks were preserved (Milàn \& Bromley, 2006), i.e. if they are preserved as undertracks. In the case of the tracksites of Highway A16, we can rule out this factor as the excavations were carried out level-by-level, so the footprints are true tracks (or natural casts). As the foot-substrate interaction is a major determinant of the final shape of a track, it is important to analyse variations in depth and shape along trackways to ascertain the morphological variation (e.g.: Razzolini et al., 2014). For this reason, we first analysed the individual footprint shape (Figs. 2, 3) and then looked at the variation along the trackway (Figs. 4, 5). The idea was to establish whether some of the described morphotypes might represent variations produced by the same/similar trackmakers (in the sense of a theropod with tridactyl functionally similar pes structure, as it is not possible to assign the tracks to a particular clade) walking on a substrate with different properties (water content, thickness or cohesiveness). Previous researchers have described variations in dinosaur footprint shape between two extremes of a morphological continuum or a gradational series (Gatesy et al., 1999; Razzolini et al., 2014) to suggest that similar theropods traversed substrates of variable consistency. Other researchers have shown variations in dinosaur footprint morphology as a consequence of locomotor adaptations associated with changes in substrate consistency (Wilson et al., 2009). Thus, the same trackmaker can produce footprints with significant shape variation along the trackways when there is a change in the aforementioned substrate properties. Only in such cases where the analysis is along the trackway, the differences can be claimed as a consequence of foot-substrate interactions rather than anatomical differences in the foot morphology of the trackmaker. In the Swiss samples, clear evidence of intermediate morphologies is missing, supporting the presence of at least two different groups of tridactyl 
290

291

292

293

294

295

296

297

298

299

300

301

302

303

304

305

306

307

308

309

310

311

312

313

314

315

316

317

318

319

320

321

322

323

324

325

326

327

328

trackmakers. Where gradational series of theropod tracks have been reported (see refs above), these show a hallux, metatarsal marks, and distinctive displacement rims in the deepest tracks that are clearly extramorphological features. None of the morphotypes presented in this paper shows such evidence, even in the deepest tracks. This leads us to think that the sediment was relatively firm during the production of the tracks.

Generally, tracks with a preservation grade of 1 or higher can be classified in one of the two described morphotypes: gracile or robust. There are just a few classification doubts regarding isolated footprints (e.g.: CRO500-T30BIS-R4). At the outset, one possible hypothesis was that the robust morphotype could be a variation of the gracile morphotype, produced by a similar trackmaker on a substrate with different rheological properties (e.g.: Gatesy et al., 1999; Razzolini et al., 2014). This hypothesis was especially appealing given the similar footprint dimensions of the two morphotypes. Thus, the deeper tracks would look more robust than the shallow ones, and the absence of clear phalangeal pad marks in most of the robust morphotype tracks might be a consequence of a softer substrate or of deeper penetration by the trackmaker foot. Our max-depth analysis (see Tables 2 and S1), indeed showed that the robust tracks have high values of maximum depth (e.g.: BEB500-T120-R5 = 6.1 mm; BEB500-T120-R6 = $10 \mathrm{~mm}$; BEB500-E1 = $10.5 \mathrm{~mm}$; TCH1065-E124 = $6.9 \mathrm{~mm}$; TCH1065-E188 = 5.9 mm; TCH1065-T15$\mathrm{R} 1=8.3 \mathrm{~mm}$; TCH1065-T21-R1 = $12.1 \mathrm{~mm}$, see Table S1). However, it is worth noticing that the highest values all occur on level TCH1065, where also the gracile tracks show their deeper values $($ TCH1065-E28 $=11.7 \mathrm{~mm}$; TCH1065-T25-R2 $=12.9 \mathrm{~mm}$; TCH1065-T25-L2 = 10.2 $\mathrm{mm}$ ), quite comparable to the robust ones. Therefore, on this track level the presence of the two morphotypes cannot be directly associated with the depth of the footprints, as both the gracile and the robust show similar values of maximum depth. In the case of BEB500 we see a similar scenario, e.g., BEB500-T16 and BEB500-T17 (gracile) have the same depth as BEB500-T120 and BEB500-E1 (robust). In other words, the depth of the tracks is more determined by the level where they were impressed than by being robust or gracile. Anyway, it is noteworthy that the max-depth analysis shows that depth values are relatively low with maximum depths of slightly more than just $1 \mathrm{~cm}$ in the deepest tracks.

The analysis of the morphological variation along the trackways shows that the gracile morphotype is quite consistent along the trackways, and no tracks classifiable as robust are found within these trackways. There are only a few cases, e.g. CRO500-T30BIS-R4 (Fig. 5B) and BEB500-T17-L8/ BEB500-T17-L9 (Fig. 4B), which might look more robust than the other tracks in the trackway, but here the features did not properly fit with the description of the robust morphotype. Regarding the robust morphotype, in the analysed trackways (BEB500-T120, TCH1065-T15 and TCH1069-T2) none of the tracks shows any feature of the gracile morphotype (noteworthy is the low preservation grade and the scarce data for TCH1065-T15 and TCH1069-T2). The maximum variation in depth along the trackways is low, being around $5 \mathrm{~mm}$ in both morphotypes (Gracile, CRO500-T30BIS = $4.7 \mathrm{~mm}$; Robust, BEB500-T120 = $5.8 \mathrm{~mm}$ ).

These data suggest that, in our case, there is no clear correlation between the depth of the 
footprint and the morphotypes (either gracile or robust), and that the intra-trackway variation is never significant enough to denote a shift between the morphotypes. Therefore, the present evidence indicates that there are at least (see following discussion) two different trackmakers of small to medium-sized theropods in the tidal flats of the Jura Mountains.

Analysis of the mesaxony (it represents how far the projection of digit III extends with respect to digits II and IV) and the FL/FW ratio supports the presence of at least the two morphotypes (Fig. 6). Some authors have used mesaxony (Weems, 1992; Lockley, 2009) as a good parameter to distinguish between tridactyl tracks. In the studied sample, this parameter is clearly lower in the robust morphotype than in the gracile one. The FL/FW ratio also shows a considerable difference between the morphotypes (likewise lower in the robust morphotype). A closer look at these two parameters within the robust morphotype (Fig. 6B) raises the question whether it represents a single ichnotaxon. The data for the two analysed tracks from BEB500-T120 (AT = 0.38-0.40; $\mathrm{FL} / \mathrm{FW}=1.13-1.16$ ) show considerably lower data for the FL/FW ratio and weaker mesaxony than the tracks from TCH1065, $(\mathrm{AT}=0.52-0.61 ; \mathrm{FL} / \mathrm{FW}=1.23-1.46)$ (see also following discussion).

2) Morphotype variation due to ontogeny?

Another salient point relating to the number of morphotypes in the analysed sample is the possibility of variations due to different ontogenetic states. Few works have dealt with the relationship between dinosaur footprints and ontogeny (e.g.: Lockley, 1994; Matsukawa, Lockley \& Hunt, 1999; Hornung et al., 2016). Ontogenetic variations have been suggested to explain morphological variation in the classical theropod ichnotaxa of the Grallator-Eubrontes plexus (Olsen, 1980; Olsen, Smith \& McDonald, 1998; Moreau et al., 2012). Olsen, Smith \& McDonald, (1998) proposed that the major proportional differences between Grallator, Anchisauripus and Eubrontes might be derived from the allometric growth of individuals of several related species. In these typical theropod footprints the large tracks (Eubrontes) are wider with weaker mesaxony than the smaller tracks (Grallator), showing a positive correlation between the elongation of the track and the elongation of the anterior triangle (Lockley, 2009). As this author suggested, the assumption of ontogenetic variation is thus based mainly on the assumption of a discernible allometric pattern. Although the growth dynamics have been documented in several groups of theropods (Bybee et al., 2006; Griffin, 2018 and references therein) little is known about how possible ontogenetic variations may have affected variations in theropod feet proportions and thus in footprint shape (Farlow and Lockley, 1993; Farlow et al., 2013). Generally tracks that are similar in morphology but different in size are considered to belong to the same ichnotaxon (Thulborn, 1990; Lockley, 1994; Matsukawa, Lockley \& Hunt, 1999; Clark, Ross \& Booth, 2005; Pascual-Arribas \& Hernández-Medrano, 2011; Castanera et al., 2015). Demathieu (1990) also explored the use of ratios of length characters to reduce the influence of size when comparing footprints. For instance, Lockley, Mitchel \& Odier (2007) assumed that small theropod tracks (Carmelopodus) from the Jurassic of North America represent adults of small species and not juveniles of larger species and suggested that "this 
368

369

370

371

372

373

374

375

376

377

378

379

380

381

382

383

384

385

386

387

388

389

390

391

392

393

394

395

396

397

398

399

400

401

402

403

404

405

406

inference is consistent with a model of rapid growth rates such as is typical of birds, which would have reduced the number of potential track making juveniles that could habitually make footprints". By contrast, Pascual Arribas and Hernández-Medrano (2011) considered minute theropod tracks from the Early Cretaceous of Spain (subsequently assigned to Kalohipus bretunensis by Castanera et al., 2015) to belong to baby theropods because of the morphometric similarities with larger tracks from the same site and formation.

Two possible different ontogenetic stages should be considered in the interpretation of the Ajoie ichnocoenosis. In one case, there are similarities between the gracile morphotype and the previously described Carmelopodus tracks from the Chevenez-Combe Ronde tracksite (CRO500-T8; CRO500-T10; CRO500-T16; CRO500-T21; CRO500-T26; CRO500-T41) so the first hypothesis would be that both are an ontogenetic variation of the same morphotype. According to the original description by Marty (2008), these tracks can be characterized as mesaxonic, slightly asymmetric, tridactyl tracks that are clearly longer than wide. Digit III is always the longest, digit IV being longer than digit II, which is shorter posteriorly. Claw impressions are present in the three digits, and there is a phalangeal pad formula of 2-3-3. There is a low total divarication angle, and divarication angles of the same order between digits II and III, and III and IV. It has a narrow-gauge trackway with small tracks with outward rotation. CRO500-T10-L10 is the track with the highest preservation grade recovered from level CRO500. Regarding the data taken from this footprint, it should be noted that the FL/FW ratio (1.69) falls within the range of the other gracile tracks, while the mesaxony is among the highest in the whole sample (0.96) but still within the range of the gracile morphotype (Fig. 6). The divarication angle is also low $\left(32^{\circ}-23^{\circ}\right)$. Moreover, reanalysis of the tracks with the use of falsecolour depth maps (Fig. 2F) allowed the fourth phalangeal pad in digit IV to be distinguished, suggesting a formula of 2-3-4, although this is not preserved in most of the tracks with a lower preservation grade (Fig. 5A). Accordingly, we consider that there are not enough data to interpret CRO500-T10-L10 as a different morphotype of the tracks included in the gracile morphotype and we regard them as part of it (see next section for the new ichnotaxonomic assignation). This highlights the importance of analysing large samples and the variation in shape through the trackways. The differences in size between CRO500-T10-L10 $(\mathrm{FL}=11 \mathrm{~cm})$ and the rest of the specimens of the gracile morphotype $(\mathrm{FL}=16-21.2 \mathrm{~cm})$ but similar footprint shape, $\mathrm{FL} / \mathrm{FW}$ ratio and mesaxony might be explained by an isometric growth. However, the absence of more data of smaller size classes of the gracile morphotype, prevent us to test this first hypothesis.

A second hypothesis considers whether the gracile and the robust morphotype might be ontogenetic variations of one of the three previously identified larger ichnotaxa (Megalosauripus transjuranicus, Jurabrontes curtedulensis and the informally named Morphotype II tracks) of the Jura Mountains (Razzolini et al., 2017; Marty et al., 2017). The two formally described ichnospecies represent large and more slender (Megalosauripus transjuranicus) and giant and more robust (Jurabrontes curtedulensis) theropod tracks, respectively. In addition, the third large morphotype not assigned to any known ichnotaxon and named Morphotype II is characterized by 
407

408

409

410

411

412

413

414

415

416

417

418

419

420

421

422

423

424

425

426

427

428

429

430

431

432

433

434

435

436

437

438

439

440

441

442

443

444

445

subsymmetric tracks that are generally slightly longer than wide (sometimes almost as wide as long), blunt digit impressions, with no evidence for discrete phalangeal pad and claw marks (Razzolini et al., 2017). The interpretation of this morphotype is quite complex, as tracks with the aforementioned features have been also documented as a morphological variation in Megalosauripus trackways. However, other trackways show a very consistent morphology throughout very long trackways, and have been considered by the authors a true unnamed ichnotaxon different from Megalosauripus and with a probable ornithopod affinity. This means that tracks with Morphotype II features can represent two different trackmakers, a theropod (Megalosauripus) and an ornithopod (the proper informally named Morphotype II). Long trackways of Morphotype II are found on the same surfaces that many in the studied sample come from, such as BEB500 and CRO500 (Razzolini et al., 2017). Thus, the hypothesis that the gracile and the robust morphotypes might represent juvenile/subadult specimens of the larger tracks described in the tracksites must be explored.

Analysing footprint proportions, it should be noted that the FL/FW ratio of the gracile morphotype fits within the upper range of the tracks included in the ichnotaxon Megalosauripus (Fig. 6A) from the Reuchenette Formation; considering just the type material of Megalosauripus transjuranicus, it fits well (Fig. 6B) (Razzolini et al., 2017). However, the mesaxony is substantially higher in the gracile morphotype than in the Megalosauripus tracks. In the case of the robust morphotype, the FL/FW ratio fits within the range of Jurabrontes curtedulensis and the Morphotype II tracks when analysing all the referred material (Fig. 6A) or just the type material of Jurabrontes curtedulensis and the best-preserved tracks of Morphotype II (BEB500TR7-L2; BEB500-TR7-R2; BEB500-TR7-R7; BEB500-TR7-L10, Razzolini et al., 2017) (Fig. $6 \mathrm{~B})$. The robust morphotype has higher mesaxony than Jurabrontes curtedulensis, being more similar in this respect to the Morphotype II tracks. It is notable that the footprint proportions within the robust morphotype are quite variable between stratigraphic levels. For example, tracks from trackway BEB500-T120 have a lower FL/FW ratio and mesaxony, whereas tracks from track level TCH1065 have higher ratios. Thus, BEB500-T120 is closer to the ranges of Jurabrontes curtedulensis whereas the tracks from TCH1065 are closer to the ranges of Megalosauripus transjuranicus and especially the Morphotype II tracks (Fig. 6).

As previously discussed, the variations in mesaxony, where larger tracks have lower mesaxony, are well documented in theropod tracks (Weems, 1992; Olsen, Smith \& McDonald, 1998; Lockley, 2009). Because there are some overlapping areas in the footprint proportions of the larger and the smaller tracks, it might be tempting to relate them according to these values; i.e. gracile with $M$. transjuranicus, robust from BEB500 with Jurabrontes, and robust from TCH1065 with Morphotype II. Nonetheless, the smaller morphotypes show other considerable morphological differences apart from size and mesaxony with respect to the larger morphotypes. The gracile morphotype differs from M. transjuranicus in key features of the diagnosis such as the sigmoidal impression of digit III (less sigmoidal), the divarication angle (less divaricated) and the digital pad of digit IV (proportionally smaller when preserved). The robust morphotype 
446

447

448

449

450

451

452

453

454

455

456

457

458

459

460

461

462

463

464

465

466

467

468

469

470

471

472

473

474

475

476

477

478

479

480

481

482

483

484

485

(from both BEB500 and TCH1065) differs from Jurabrontes curtedulensis in the absence of clear phalangeal pads (preservation bias?), the absence of the peculiar, isolated proximal pad PIII1 of digit III, and the interdigital divarication angles (asymmetric vs symmetric); it also differs from the Morphotype II tracks in the absence of blunt digit impressions, possible evidence of a discrete phalangeal pad, and the presence of clear claw marks. Therefore, despite some overlap of the morphometric data and the fact that there are considerable differences in shape (although some of them might be extramorphological variations), we consider that there are not enough data to support the second hypothesis just on the basis of the footprint shape and morphometric data.

Finally, we examine whether there is any spatio-temporal relationship between the larger and the smaller tracks from the Ajoie ichnocoenosis. Lockley (1994) warned that the track data "that most probably represent monospecific assemblages are those obtained for a single ichnotaxon from a single bedding plane". In this regard, it is interesting to note the scarcity of large theropod tracks in the ichnoassemblages where both the gracile and the robust morphotype have been identified, mainly levels BEB500, TCH1065 and CRO500. Level BEB500 (Fig. S1), the one with the highest number of studied tracks $(n=39)$, is mainly composed of sauropods $(n=17$ trackways) and minute to small tridactyl ( $\mathrm{n}=158$ trackways $)$ tracks. No tracks assigned to Jurabrontes curtedulensis or M. transjuranicus have been documented in this level although it is the surface with the most Morphotype II tracks ( $\mathrm{n}=8$ trackways) documented. Level TCH1065 (Fig. S2) ( $\mathrm{n}=15$ studied tracks) is composed of 189 tracks, mainly of minute to small-sized theropods, and two parallel trackways (TCH1065-T26, TCH1065-T27) assigned to Jurabrontes have also been documented. In level CRO500 (Fig. S3) (n = 20 studied tracks), 16 sauropod trackways and 57 tridactyl trackways have been documented. One of the tridactyl trackways (CRO500-T43) has been assigned to Morphotype II (Razzolini et al., 2017). Thus, there are in three cases a large track type (Morphotype II in BEB500 and CRO500, and Jurabrontes in TCH1065) and the robust and the gracile morphotypes in the same surface (Figs. S1-S3). Interestingly, no Megalosauripus tracks have been documented in any of the three levels. One way to confirm that some of the small tracks were juveniles of the larger ichnospecies would be to find some kind of relationship among them, such as gregarious behaviour (sensu Castanera et al., 2014). However, there is no clear relationship between the trackways, neither of the same size nor of different sizes. In BEB500 (Fig. S1), trackways TR1, TR3, TR4, TR5, TR6 and TR8 (Morphotype II) cross several trackways made by small trackmakers, but the orientations are completely different and do not show any kind of relationship. TR2 (Morphotype II) is subparallel with T34 (small track but unknown morphotype) at the beginning of the trackway but shows a significant change in direction, so this does not show any relationship either. Notably, TR7 (Morphotype II) is a long trackway that is subparallel to T120 (robust morphotype). Tracks T120-L10 and T120-R10 tread over tracks TR7-R8 and TR7-L9 but pass afterwards, so although this might indicate some kind of interaction there is no clear evidence of gregarious behaviour. In level TCH1065 (Fig. S2), the two parallel trackways (TCH1065-T26, TCH1065-T27) assigned to Jurabrontes do not show any evidence of a relationship with the smaller tracks either. Finally, 
486 in CRO500 (Fig. S3), T43 (Morphotype II) is slightly subparallel to T42 (small track but 487 unknown morphotype), but there is no clear evidence to suggest that they were walking together. 488 To sum up, generally the orientation of the large trackways does not seem to suggest any sort of 489 relationship, with the possible exception of TR7 and T120. This single case might hint at the 490 hypothesis that some tracks of the robust morphotype (BEB500-T120) might represent a juvenile 491 of the producer of the tracks classified as Morphotype II. However, BEB500-T120 is the very 492 trackway that shows more morphometric similarities to Jurabrontes than to Morphotype II (Fig. 493 6), thus weakening this hypothesis. In the light of the previous discussion, there is no evidence to 494

495

496

497

498

499

500

501

502

503

504

505

506

507

508

509

510

511

512

513

514

515

516

517

518

519

520

521

522

523 suggest an interaction (i.e. behavioural aspect) among the dinosaurs that produced trackways of different sizes when they are left on the same surface. Thus, there is no indication (nor in footprint shape, nor morphometric, nor spatio-temporal) to suggest that the gracile and the robust morphotype are smaller tracks of the same of the larger morphotypes described from the area. Thus, the differences between the larger and the smaller morphotypes have thus led us to treat them as different ichnotaxa (see next section).

3) Ichnotaxonomy:

As noted by Marty (2008), small to medium-sized tridactyl tracks are generally not very common in the Late Jurassic and Early Cretaceous, and accordingly such tracks have only recently been the focus of ichnotaxonomic descriptions. Although recent descriptions have considerably increased the number of small to medium-sized tridactyl tracks, few ichnotaxa have been described in the Late Jurassic and Early Cretaceous of Europe. Lockley, Meyer and Moratalla (2000) suggested that theropod track morphologies are much more variable through time than previously thought. These authors pointed out that "the perception of morphological conservatism and uniformity through time is, in part, a function of lack of study of adequately large samples of well-preserved material (Baird, 1957)". In this sense, the studied tracks from the Ajoie ichnocoenosis represent a good sample of tridactyl dinosaur tracks in terms of the number of specimens $(n=93)$, with a considerable quality of preservation in many of them $(n=23$ with a preservation grade greater than 2 ).

Although they are not very abundant in other European tracksites, small to medium-sized tridactyl trackways are the most abundant in the Ajoie ichnocoenosis. As mentioned above, the main small to medium-sized tridactyl dinosaur ichnotaxa that have been described from the Late Jurassic of Europe are (Fig. 7) Grallator (Fig. 7A) and Anomoepus (Fig. 7B) in Spain (Lockley et al., 2008; Piñuela, 2015; Castanera, Piñuela \& García-Ramos, 2016); Carmelopodus (Fig. 7C) and Eubrontes (Fig. 7D) in France (Mazin et al., 2000; Mazin, Hantzpergue \& Pouech, 2016); Wildeichnus isp. (Fig. 7E), cf. Jialingpus (Fig. 7F) and Dineichnus (Fig. 7G) in Poland (Gierliński, Niedźwiedzki \& Nowacki, 2009); Dineichnus (Fig. 7H) (Lockley et al., 1998a) and Therangospodus-like tracks (Fig. 7I) (Lockley, Meyer \& Moratalla, 2000) in Portugal; and Grallator in Germany (Fig. 7J) (Diedrich, 2011). In addition, Conti et al. (2005) described medium-sized footprints (Fig. 7K) that "resemble Therangospodus" (their type 3) and another 
524 morphotype (their type 2, based on three specimens, Fig. 7L) that shares the same functional 525 character with Carmelopodus, i.e., the lack of the fourth proximal pad on digit IV.

526 When compared with the type specimens of these ichnotaxa, the new data on the gracile morphotype of CRO500-T10 (Figs. 5A; 8N) (see previous sections) allow us to rule out the

528

529

530

531

532

533

534

535

536

537

538

539

540

541

542

543

544

545

546

547

548

549

550

551

552

553

554

555

556

557

558

559

560

561

562 presence of Carmelopodus untermannorum (Fig. 8A) in the Ajoie, as previously discussed. Generally, the gracile morphotype (Fig. 8M-8O) does not fit with key features of the diagnosis of this ichnotaxon (Lockley et al., 1998b), differing in the phalangeal pad formula (2-3-4 rather than 2-3-3), symmetry, different length/width ratio, or the lower divarication. Among other theropod ichnotaxa, the gracile morphotype shows considerable differences with respect to Wildeichnus navesi (Fig. 8B, Casamiquela, 1964; Valais, 2011) from the Jurassic of Argentina (as well as larger size, a not subequal but lower divarication angle, larger claw marks, an unrounded digital phalangeal pad in digit IV, greater asymmetry, a generally higher length/width ratio); and with respect to Therangospodus pandemicus from the Late Jurassic of North America and Asia (Fig. 8C, smaller size, presence of clear phalangeal pads, higher mesaxony) (Lockley, Meyer \& Moratalla, 2000; Fanti et al., 2013). The differences with respect to ornithopod ichnotaxa are noteworthy: it differs from Anomoepus scambus (Fig. 8D) in being less symmetric, having a metatarsal-phalangeal pad of digit IV not in line with the digit III axis, no hallux marks, higher mesaxony, and no manus prints present (see Olsen \& Rainforth, 2003; Piñuela, 2015). It also differs notably with respect to Dineichnus socialis (Fig. 8E) for higher FL/FW ratio, higher mesaxony, no quadripartite morphology, a different heel pad impression, lower digit divarication (see Lockley et al., 1998a).

The features of the gracile morphotype fit better with the smaller ichnotaxa of the GrallatorAnchisauripus-Eubrontes (Fig. 8F-8H) plexus (Olsen, 1980; Demathieu, 1990; Weems, 1992; Olsen, Smith \& McDonald, 1998): small to medium-sized, well-defined digital pads, digits II and IV of similar length, digit III being longer and showing high mesaxony, an oval/subrounded "heel" and a low interdigital angle. Although these footprints have mainly been described from Late Triassic and Early-Middle Jurassic deposits, in recent years they are also known from younger strata including the Late Jurassic of Europe (see Castanera, Piñuela \& García-Ramos, 2016 and references therein). Regarding the use of the ichnotaxon Anchisauripus, Castanera, Piñuela \& García-Ramos (2016) wrote a short review examining how different authors have considered Grallator and Anchisauripus as synonyms (Lucas et al., 2006; Lockley, 2009; Piñuela, 2015). The main sample of "grallatorid" tracks that has been described from Late Jurassic deposits in Europe comes from Asturias (Spain), and these have been assigned to Grallator (Castanera, Piñuela \& García-Ramos, 2016). However, the gracile tracks from the Ajoie ichnocoenosis differ in the digit proportions (FL/FW ratio) and mesaxony (Fig. 9) from those in Asturias. It should be noted that the Asturian sample shows a great variation in mesaxony (that does not correlate with size). This holds also true for the gracile morphotype although the footprint proportions are less variable. Whereas Castanera, Piñuela \& GarcíaRamos (2016) stated that mesaxony "should be used with caution in distinguishing between 
563 different ichnotaxa", we consider that the differences in mesaxony between the gracile 564 morphotype (AT $=0.53-0.98)$ and the Grallator tracks from Asturias (AT $=0.72-1.12$, Castanera, 565 Piñuela \& García-Ramos 2016) tracks are large enough to do so. Furthermore, the FL/FW ratio 566 is also considerably higher in the Grallator tracks (FL/FW ratio $=1.73-2.5$, Castanera, Piñuela \& 567 García-Ramos 2016) than in the gracile morphotype (FL/FW ratio $=1.50-1.90)$. Oversplitting 568 has occurred in some theropod ichnotaxa similar to Grallator-Eubrontes plexus. For example, 569 Lockley et al. (2013) proposed a great reduction in the Jurassic theropod ichnotaxa from Asia, 570 arguing that many of them were subjective junior synonyms of Grallator and Eubrontes. 571 Nonetheless, the authors retain the ichnotaxon Jialingpus yuechiensis (Fig. 8I) from the Late 572 Jurassic-Early Cretaceous of China (Xing et al., 2014). On the basis of digit proportions (FL/FW 573 ratio) and mesaxony, the gracile morphotype falls partially within the range of Jialingpus but also within the range of Kalohipus bretunensis (Fig. 8J) from the Early Cretaceous (Berriasian) of Spain (Fuentes Vidarte \& Meijide Calvo, 1998; Castanera et al., 2015). According to Xing et al. (2014), the main differences between Jialingpus and Grallator are the presence of a digit I trace and the large metatarsophalangeal area positioned in line with digit III, which are its main features. These features are absent in the gracile morphotype, so an assignment to Jialingpus can be excluded. On the other hand, the diagnosis of Kalohipus bretunensis (Fuentes Vidarte \& Meijide Calvo, 1998) clearly includes features that distinguish it from the gracile morphotype, such as its smaller size or robust digits, and as seen in Fig. 9, the footprint proportions and especially the mesaxony are also slightly different. As mentioned above, the morphology is also different from the larger ichnotaxa (Jurabrontes curtedulensis, Fig. 8K, and Megalosauripus transjuranicus, Fig. 8L) that occur in the same deposits.

To summarize, the gracile morphotype is quite similar to other grallatorid tracks (Grallator, Anchisauripus, Kalohipus, Jialingpus), the main differences being the digit proportions and mesaxony. Given the current state of knowledge, it is difficult to interpret how much variation between the aforementioned ichnotaxa is a consequence of variations in preservation, ontogeny or ichnodiversity. Taking into account the whole discussion, and bearing in mind the high variation in both the FL/FW ratio and mesaxony seen in tracks assigned to Grallator, we thus tentatively classify the gracile morphotype as cf. Kalohipus isp., as this is the ichnotaxon that is closest to it (Fig. 9). Future studies should elucidate the similarities and differences between these grallatorid tracks. Jialingpus tracks have been also described in the Late Jurassic/Early Cretaceous of Europe (Gierliński, Niedźwiedzki \& Nowacki, 2009), and an analysis of the differences between Jialingpus and other grallatorid tracks (including Kalohipus) is "pending" (Xing et al., 2014). In this regard, it would be interesting to note the differences in mesaxony among both Kalohipus and Jialingpus (low mesaxony) and Grallator (high mesaxony), and questioning whether mesaxony is a good measure for discriminating between the three ichnotaxa. Possible substrate related differences in preservation or ichnofacies substrates have to be tested too. For example, Kalohipus bretunensis and the main grallatorid ichnotaxa (Fig. 8F-J) Mountains are preserved in marginal marine carbonates. 
603 Regarding the robust morphotype (Fig. 8P-8Q), a crucial question is whether it represents a 604 single ichnotaxon. In this context, it should be noted that, the morphology of the tracks with a 605 preservation grade of 2 or more, as well as the footprint proportions (Fig. 6B) such as those of 606 trackway BEB500-T120 and the tracks from TCH1065 (TCH1065-T21-R1, TCH1065-E124 and 607 TCH1065-E188) varies considerably. The appearance of this morphotype is completely different 608 from ichnotaxa like Carmelopodus untermannorum (Fig. 8A, size, phalangeal pad formula, digit 609 divarication, well-developed claw marks), Wildeichnus navesi (Fig. 8B, size, gracility, 610 symmetry, length/width ratio and mesaxony), Anomoepus scambus (Fig. 8D, size, absence of a 611 manus impression, morphology of the metatarsal-phalangeal pad of digit IV) and Dineichnus 612 socialis (Fig. 8E, no quadripartite morphology or circular heel pad impression). It differs also from all the aforementioned grallatorid ichnotaxa Grallator-Anchisauripus-Eubrontes, plus Jialingpus, Kalohipus (Fig. 8F-J, mainly in the more robust morphology, footprint proportions, mesaxony, heel morphology, divarication) and the larger ichnotaxa (Jurabrontes curtedulensis, Fig. 8K, and Megalosauripus transjuranicus, Fig. 8L) that occur at the same localities..

617 Of all the known ichnotaxa, it shares most similarities with Therangopodus pandemicus (Fig. 8C, 618 Lockley, Meyer \& Moratalla, 2000; Fanti et al., 2013; see also Castanera et al., 2013 for new 619 data on tracks previously assigned to Therangospodus), although the robust morphotype from the 620 Swiss Jura Mountains has a higher digit divarication and probably higher mesaxony 621 (unpublished data for this parameter in the original publication, Lockley, Meyer \& Moratalla, 2000). According to the original diagnosis, this ichnotaxon is a "medium sized, elongate, asymmetric theropod track with coalesced, elongate, oval digital pads, not separated into discrete phalangeal pads". Trackway narrow with little or no rotation of digit III long axis from trackway axis". The tracks from the Ajoie ichnoassemblages are slightly smaller in size than Therangospodus pandemicus (Lockley, Meyer \& Moratalla, 2000; Fanti et al., 2013). According to these authors, and based on the original descriptions by Lockley, Meyer \& Moratalla (2000),

628 Therangospodus is characterized by: "1) oval digital pads not separated into discrete digital pads, 2) no rotation of digit III, 3) narrow trackway, and 4) relatively reduced size ( $<30 \mathrm{~cm}$ in average length)". Regarding the absence of discrete digital pads, Lockley, Meyer \& Moratalla (2000) described in the type ichnospecies of Therangospodus the presence of "faint indentations at the margin of the pads" that sometimes reveal the location of the phalangeal pads, suggesting a 2-3-4 phalangeal pad formula. Razzolini et al. (2017) also pointed out the difficulties of distinguishing between Therangospodus and Megalosauripus. This has also been previously discussed by other authors (Gierliński, Niedźwiedzki \& Pieńkowski, 2001; Piñuela, 2015), suggesting that some of the diagnostic features might be extramorphological variations. It is notable that Megalosauripus and Therangospodus generally co-occur in the same sites (Meyer \& Lockley, 1997; Lockley, 638 Meyer \& Moratalla, 2000; Lockley, Meyer \& Santos, 2000; Xing, Harris \& Gierliński, 2011; 639 Fanti et al., 2013), which might be relevant as the size and preservation could be the only 640 differences between the two ichnotaxa. Interestingly, as we have seen in the previous section, the 641 robust morphotype does not co-occur with any Megalosauripus tracks, although some of them 642 (BEB500-T120) co-occur with tracks described as Morphotype II. Even though the robust 
643 morphotype is reminiscent of Therangospodus pandemicus, it is not possible to assign it to this 644 ichnospecies or to any of the known small-medium-sized ichnotaxa. The rarity of collected 645 specimens and the preservation grade (none of them as high as 2.5-3) prevents us from erecting a 646 new ichnotaxon. Taking into account that Therangospodus pandemicus is the closest ichnotaxon 647 described, we thus tentatively classify the tracks from level TCH1065 as cf. Therangospodus isp. 648 and the tracks from BEB500 as Therangospodus? isp. in order to show that there are some 649 differences (both morphometric and in shape) within the robust morphotype. As for the Swiss 650 specimens, Therangospodus pandemicus tracks have been preserved in carbonate materials 651 (Lockley, Meyer \& Moratalla, 2000), so we can rule out the differences between this ichnotaxon 652 and the robust morphotype being a consequence of this factor as the substrate and the 653 palaeonvironmental conditions were probably similar at the time of track production.

\section{5}

656

657

658

659

660

661

662

663

664

665

666

667

668

669

670

671

672

673

674

675

676

677

678

679

680

681

\section{CONCLUSIONS}

The minute to medium-sized tridactyl dinosaur footprints from the tracksites of Highway A16 in the Jura Mountains (NW Switzerland) represent one of the largest samples from the Late Jurassic worldwide. The integrated analyses of the quality of preservation (preservation grade), the maximum depth, the shape variation along the trackway, and the footprint proportions (FL/FW ratio and mesaxony) open a new window into the interpretation of dinosaur track variations also considering other factors such as preservation, ontogeny and ichnotaxonomy. The descriptions and analyses of the material have made it possible to characterize in detail two different morphotypes, one gracile and one robust, that were already identified in the field. The new data allow us to rule out the notion that the two morphotypes represent a morphological continuum of extramorphological variations, or ontogenetic variations of the larger tracks described from the same sites. New morphometric data allow us to include the small sized tracks previously described from Chevenez-Combe Ronde tracksite within the gracile morphotype, being the only case in the studied sample that might be explained by an ontogenetic variation. An ichnotaxonomical comparison with the main minute to medium-sized tridactyl ichnotaxa did not allow assigning them to any known ichnotaxon with confidence. The gracile morphotype, though similar to some grallatorid ichnotaxa, shows a number of morphometric differences and have been assigned to cf. Kalohipus isp. The robust morphotype, though similar to Therangospodus pandemicus, also shows some differences with respect to the diagnosis of the type specimen, and therefore is classified as cf. Therangospodus isp. and Therangospodus? isp. Further work is needed in order to understand the possible influence of the substrate composition on theropod ichnotaxonomy in general and the aforementioned ichnotaxa in particular. This study also highlights the difficulties of distinguishing between minute and medium-sized tridactyl dinosaur ichnotaxa and the importance of analysing different factors related to preservation and ontogeny before assigning a single track to a specific ichnotaxon. The new data increase theropod ichnodiversity to $4 / 5$ ? theropod ichnotaxa in the tidal flats of the Jura carbonate platform and support previous suggestions that carbonate tidal flats were mainly dominated by theropod and sauropod dinosaurs (Lockley,Hunt \& Meyer 1994; D’Orazi Porchetti et al., 2016). 
REFERENCES

684 Alcalá L, Mampel L, Royo-Torres R, Cobos A. 2014. On small quadrupedal ornithopod tracks in 685 Jurassic-Cretaceous transition intertidal deposits (El Castellar, Teruel, Spain). Spanish Journal of 686 Palaeontology 29 (2):83-190.

687 Antunes MT, Mateus O. 2003. Dinosaurs of Portugal. Comptes Rendus Palevol 2(1): 77-95.

688 Baird D. 1957. Triassic reptile footprint faunules from Milford, New Jersey. Bulletin of The 689 Museum of Comparative Zoology 117:449-520.

690 Belvedere M, Mietto P, Ishigaki S. 2010. A Late Jurassic diverse ichnocoenosis from the 691 siliciclastic Iouaridene Formation (Central High Atlas, Morocco). Geological Quarterly 692 54(3):367-380.

693 Belvedere M, Farlow JO. 2016. A numerical scale for quantifying the quality of preservation of 694 vertebrate tracks. In: Falkingham PL, Marty D, Richter A, eds. Dinosaur Tracks-The next 695 steps. Bloomington and Idianapolis: Indiana University Press. 92-99.

696 Bybee PJ, Lee AH, Lamm ET. 2006. Sizing the Jurassic theropod dinosaur Allosaurus: assessing 697 growth strategy and evolution of ontogenetic scaling of limbs. Journal of Morphology 267(3): $698 \quad 347-359$.

699 Campos-Soto S, Cobos A, Caus E, Benito MI, Fernández-Labrador L, Suárez-Gonzalez P., 700 Quijada E, Royo-Torres R, Mas R, Alcalá L. 2017. Jurassic Coastal Park: A great diversity of 701 palaeoenvironments for the dinosaurs of the Villar del Arzobispo Formation (Teruel, eastern 702 Spain). Palaeogeography, Palaeoclimatology, Palaeoecology 485: 154-177.

703 Casamiquela RM. 1964. Estudios icnológicos: problemas y métodos de la icnología con 704 aplicación al estudio de pisadas mesozoicas, Reptilia, Mammalia de la Patagonia. Librart.

705 Castanera D, Vila B, Razzolini NL, Falkingham PL, Canudo JI, Manning PL, Galobart À. 2013a. 706 Manus track preservation bias as a key factor for assessing trackmaker identity and 707 quadrupedalism in basal ornithopods. PLOS ONE 8(1), e54177.

708 Castanera D, Pascual C, Razzolini NL, Vila B, Barco JL, Canudo JI. 2013b.Discriminating 709 between medium-sized tridactyl trackmakers: tracking ornithopod tracks in the base of the 710 Cretaceous (Berriasian, Spain). PLOS ONE 8(11) e81830. doi: 10.1371/journal.pone.0081830.

711 Castanera D, Colmenar J, Sauqué V, Canudo JI. 2015. Geometric morphometric analysis applied 712 to theropod tracks from the Lower Cretaceous (Berriasian) of Spain. Palaeontology 58(1), 183713200. 
714 Castanera D, Piñuela L, García-Ramos JC. 2016. Grallator theropod tracks from the Late 715 Jurassic of Asturias (Spain): ichnotaxonomic implications. Spanish Journal of Palaeontology 716 31(2): 283-296.

717 Castanera D, Vila B, Razzolini NL, Santos VF, Pascual C, Canudo JI. 2014. Sauropod trackways 718 of the Iberian Peninsula: palaeoetological and palaeoenvironmental implications. Journal of 719 Iberian Geology 40(1): 49-59.

720 Clark ND, Ross DA, Booth P. 2005. Dinosaur tracks from the Kilmaluag Formation (Bathonian, 721 Middle Jurassic) of Score Bay, Isle of Skye, Scotland, UK. Ichnos 12(2): 93-104.

722 Comment G, Ayer J, Becker D. 2011. Deux nouveaux membres lithostratigraphiques de la 723 Formation de Reuchenette (Kimméridgien, Ajoie, Jura suisse)-Nouvelles données géologiques et 724 paléontologiques acquises dans le cadre de la construction de l'autoroute A16 (Transjurane). 725 Swiss Bullettin for Applied Geology16: 3-24.

726 Comment G, Lefort A, Koppka J, Hantzpergue P. 2015. Le Kimméridgien d'Ajoie (Jura, Suisse): 727 lithostratigraphie et biostratigraphie de la Formation de Reuchenette. Revue de Paléobiologie 34: 728 161-194.

729 Conti MA, Morsilli M, Nicosia U, Sacchi E, Savino V, Wagensommer A, Di Maggio L, Gianolla 730 P. 2005. Jurassic dinosaur footprints from southern Italy: footprints as indicators of constraints in 731 paleogeographic interpretation. Palaios 20(6): 534-550.

732 Demathieu GR. 1990. Problems in discrimination of tridactyl dinosaur footprints, exemplified by 733 the Hettangian trackways, the Causses, France. Ichnos 1(2): 97-110.

734 Diedrich C. 2011. Upper Jurassic tidal flat megatracksites of Germany -coastal dinosaur 735 migration highways between European islands, and a review of the dinosaur footprints. 736 Palaeobiodiversity and Palaeoenvironments 91: 129-155.

737 D'Orazi Porchetti S, Bernardi M, Cinquegranelli A, Santos VF, Marty D, Petti FM, Caetano PS, 738 Wagensommer, A. 2016. A Review of the Dinosaur Track Record from Jurassic and Cretaceous 739 Shallow Marine Carbonate Depositional Environments. In: Falkingham PL, Marty D, Richter A, 740 eds. Dinosaur Tracks - The next steps. Bloomington and Idianapolis: Indiana University Press. $741 \quad 380-392$.

742 Falkingham PL. 2014. Interpreting ecology and behaviour from the vertebrate fossil track record. 743 Journal of Zoology 292(4): 222-228.

744 Fanti F, Contessi M, Nigarov A, Esenov P. 2013. New data on two large dinosaur tracksites from 745 the Upper Jurassic of Eastern Turkmenistan (Central Asia). Ichnos 20:54-71. 
746 Farlow JO, Lockley MG. 1993. An Osteometric Approach to the Identification of the Makers of 747 Early Mesozoic Tridactyl Dinosaur Footprints. In: Lucas SG, Morales M, eds. The nonmarine

748 Triassic. New Mexico Museum of Natural History and Science Bullletin, 3: 123-131

749 Farlow JO, Holtz TRJ, Worthy TH, Chapman RE. 2013. Feet of the Fierce (and Not So Fierce): 750 Pedal Proportions in Large Theropods, Other Non-Avian Dinosaurs, and Large Ground Birds. In: 751 Parrish JM, Molnar RE, Currie PJ, Koppelhus EB, eds. Tyrannosaurid Paleobiology: Indiana 752 University Press 89-132.

753 Foster JR, Lockley MG. 2006. The vertebrate ichnological record of the Morrison Formation 754 (Upper Jurassic, north America). New Mexico Museum of Natural History and Science Bullletin 755 36: 203-216.

756 Fuentes Vidarte C, Meijide Calvo M. 1998. Icnitas de dinosaurios terópodos en el Weald de 757 Soria (España). Nuevo icnogénero Kalohipus. Estudios Geológicos 54: 147-152.

758 Gatesy SM, Middleton KM, Jenkins Jr FA, Shubin NH. 1999. Three-dimensional preservation of 759 foot movements in Triassic theropod dinosaurs. Nature 399(6732): 141-144.

760 Gierliński GD, Niedźwiedzki G, Pieńkowski G. 2001. Gigantic footprint of a theropod dinosaur 761 in the Early Jurassic of Poland. Acta Palaeontologica Polonica 46(3): 441-446.

762 Gierliński GD, Niedźwiedzki G, Nowacki P. 2009. Small theropod and ornithopod footprints in 763 the Late Jurassic of Poland. Acta Geologica Polonica 59(2): 221-234.

764 Griffin CT. 2018. Developmental patterns and variation among early theropods. Journal of 765 Anatomy. doi: 10.1111/joa.12775

766 Gygi RA. 2000. Annotated index of lithostratigraphic units currently used in the Upper Jurassic 767 of Northern Switzerland. Eclogae Geologicae Helvetiae 93: 125-146.

768 Hammer Ø, Harper DAT, Ryan PD. 2001. PAST: paleontological statistics software package for 769 education and data analysis. Palaeontologia Electronica 4: 1-9.

770 Hornung JJ, Böhme A, Schlüter N, Reich M. 2016. In: Falkingham PL, Marty D, Richter A, eds. 771 Dinosaur Tracks - The next steps. Bloomington and Idianapolis: Indiana University Press. 202772225.

773 Jank M, Wetzel A, Meyer CA. 2006. A calibrated composite section for the Late Jurassic 774 Reuchenette Formation in northwestern Switzerland (?Oxfordian, Kimmeridgian sensu gallico, 775 Ajoie-Region). Eclogae Geologicae Helvetiae 99: 175-191.

776 Kaever M, de Lapparent AF. 1974. Les traces de pas de dinosaures du Jurassique de Barkhausen 777 (Basse Saxe, Allemagne). Bulletin de la Société Géologique de France 16: 516-525. 
778

779

780

781

782

783

784

785

786

787

788

789

790

791

792

793

794

795

796

797

798

799

800

801

802

803

804

805

806

807

808

809

810

Lallensack JN, Sander PM, Knötschke N, Wings O. 2015. Dinosaur tracks from the Langenberg Quarry (Late Jurassic, Germany) reconstructed with historical photogrammetry: evidence for large theropods soon after insular dwarfism. Palaeontologia Electronica 18.2.31A: 1-34

Lallensack JN, van Heteren AH, Wings O. 2016. Geometric morphometric analysis of intratrackway variability: a case study on theropod and ornithopod dinosaur trackways from Münchehagen (Lower Cretaceous, Germany). PeerJ 4, e2059.

Lange-Badré B, Dutrieux M, Feyt J, Maury G. 1996. Découverte d'empreintes de pas de dinosaures dans le Jurassique supérieur des Causses du Quercy (Lot, France).Comptes rendus de l'Académie des sciences. Série 2. Sciences de la terre et des planets 323(1): 89-96.

Lockley MG. 1994. Dinosaur ontogeny and population structure: interpretations and speculations based on fossil footprints. In: Carpenter K, Hirsch KF, Horner JR, eds. Dinosaur eggs and babies. Cambridge University Press. 347-365.

Lockley MG. 2009. New perspectives on morphological variation in tridactyl footprints: clues to widespread convergence in developmental dynamics. Geological Quarterly 53:415-432.

Lockley MG, Hunt AP, Meyer C. 1994. Vertebrate tracks and the ichnofacies concept: implications for paleoecology and palichnostratigraphy. In: Donovan, SK, ed. The Palaeobiology of Trace Fossils Wiley, 241-268.

Lockley MG, Santos VF, Meyer C, Hunt A. 1998a. A new dinosaur tracksite in the Morrison Formation, Boundary Butte, Southeastern Utah. Modern Geology 23: 317-330.

Lockley M, Hunt A, Paquette M, Bilbey SA, Hamblin A.1998b. Dinosaur tracks from the Carmel Formation, northeastern Utah: implications for Middle Jurassic paleoecology. Ichnos 5(4): 255-267.

Lockley MG, Meyer CA, Moratalla JJ. 2000. Therangospodus: trackway evidence for the widespread distribution of a Late Jurassic theropod with well-padded feet. Gaia 15:339-353.

Lockley MG, Meyer CA, Santos VF. 2000. Megalosauripus and the problematic concept of megalosaur footprints. Gaia 15:313-337.

Lockley M, Mitchell L, Odier GP. 2007. Small theropod track assemblages from Middle Jurassic eolianites of Eastern Utah: paleoecological insights from dune ichnofacies in a transgressive sequence. Ichnos 14(1-2): 131-142.

Lockley MG, Garcia-Ramos JC, Piñuela L, Avanzini M. 2008. A review of vertebrate track assemblages from the Late Jurassic of Asturias, Spain with comparative notes on coeval ichnofaunas from the western USA: implications for faunal diversity in siliciclastic facies assemblages. Oryctos 8:53-70. 
811 Lockley MG, Li JI, Li RH, Matsukawa M, Harris JD. Xing LD. 2013. A review of the tetrapod 812 track record in China, with special reference to type ichnospecies: implications for 813 ichnotaxonomy and paleobiology. Acta Geologica Sinica (English Edition) 87: 1-20.

814 Lucas SG, Klein H, Lockley MG, Spielmann JA, Gierliński GD, Hunt AP, Tanner LH. 2006. 815 Triassic-Jurassic stratigraphic distribution of the theropod footprint ichnogenus Eubrontes. New 816 Mexico Museum of Natural History and Science Bulletin 37: 86-93.

817 Mallison H, Wings O. 2014. Photogrammetry in paleontology-A practical guide. Journal of 818 Paleontological Techniques 12: 1-31.

819 Marty D. 2008. Sedimentology, taphonomy, and ichnology of Late Jurassic dinosaur tracks from 820 the Jura carbonate platform (Chevenez-Combe Ronde tracksite, NW Switzerland): insights into 821 the tidalflat palaeoenvironment and dinosaur diversity, locomotion, and palaeoecology. 822 GeoFocus 21:1-278.

823 Marty, D, Billon-Bruyat JP. 2009. Field-trip to the excavations in the Late Jurassic along the 824 future Transjurane highway near Porrentruy (Canton Jura, NW Switzerland): dinosaur tracks, 825 marine vertebrates and invertebrates. In: Billon-Bruyat JP, Marty D, Costeur L, Meyer CA, 826 Thüring, B, eds. 5th International Symposium on Lithographic Limestone and Plattenkalk 827 Abstracts and Field Guides. Société jurassienne d'émulation, actes 2009 bis. p. 94-129

828 Marty D, Meyer CA. 2012. From sauropods to cycads - The Late Jurassic terrestrial record of 829 the Swiss Jura Mountains. In: Witzmann F, Aberhan M, eds. Centen Meet Palaontologische 830 Gesellschaft - Program Abstr F Guid Terra Nostra - Schriften der GeoUnion Alfred-Wegener831 Stiftung, Potsadam. 119-120.

832 Marty D, Hug W, Iberg A, Cavin L, Meyer C, Lockley M. 2003. Preliminary Report on the 833 Courtedoux Dinosaur Tracksite from the Kimmeridgian of Switzerland. Ichnos 10: 209-219.

834 Marty D, Cavin L, Hug WA, Jordan P, Lockley MG, Meyer CA. 2004. The protection, 835 conservation and sustainable use of the Courtedoux dinosaur tracksite, Canton Jura, Switzerland. 836 Revue de Paléobiologie 9:39-49.

837 Marty D, Ayer J, Becker D, Berger JP, Billon-Bruyat JP, Braillard L, Hug WA, Meyer CA. 838 2007. Late Jurassic dinosaur tracksites of the Transjurane highway (Canton Jura, NW 839 Switzerland): overview and measures for their protection and valorisation. Bulletin für 840 Angewandte Geologie 12: 75-89

841 Marty D, Strasser A, Meyer CA. 2009. Formation and taphonomy of human footprints in 842 microbial mats of present-day tidal-flat environments: implications for the study of fossil 843 footprints. Ichnos 16(1-2):127-142. 
844 Marty D, Belvedere M, Meyer CA, Mietto P, Paratte G, Lovis C, Thüring B. 2010. Comparative 845 analysis of Late Jurassic sauropod trackways from the Jura Mountains (NW Switzerland) and the 846 central High Atlas Mountains (Morocco): implications for sauropod ichnotaxonomy. Historical 847 Biology 22: 109-133.

848 Marty D, Meyer CA, Belvedere M, Ayer J, Schafer KL. 2013. Rochefort-Les Grattes: an early 849 Tithonian dinosaur tracksite from the Canton Neuchatel, Switzerland. Revue de Paléobiologie $850 \quad 32: 373-384$.

851 Marty D, Belvedere M, Razzolini NL, Paratte G, Cattin M, Lovis C, Meyer C. 2017. The tracks 852 of giant theropods (Jurabrontes curtedulensis ichnogen. \& ichnosp. nov.) from the Late Jurassic 853 of NW Switzerland:palaeoecological and palaeogeographical implications. Historical Biology 185429.

855 Matsukawa M, Lockley MG, Hunt AP.1999. Three age groups of ornithopods inferred from 856 footprints in the mid-Cretaceous Dakota Group, eastern Colorado, North 857 America.Palaeogeography, Palaeoclimatology, Palaeoecology 147(1): 39-51.

858 Matthews N, Noble T, Breithaupt B. 2016. Close-Range Photogrammetry for 3-D Ichnology: 859 The Basics of Photogrammetric Ichnology. In: Falkingham PL, Marty D, Richter A, eds. 860 Dinosaur Tracks - The next steps. Bloomington and Idianapolis: Indiana University Press. 2886155.

862 Mazin JM, Hantzpergue P, Bassoullet JP, Lafaurie G, Vignaud P.1997. The Crayssac site (Lower 863 Tithonian, Quercy, Lot, France): discovery of dinosaur trackways in situ and first ichnological 864 results. Comptes Rendus de l'Academie des Sciences. Series IIA Earth and Planetary Science 865 9(325): 733-739.

866 Mazin JM, Billon-Bruyat JP, Hantzpergue P, Lafaurie G. 2000. Domination reptilienne dans 867 l'écosystème littoral de Crayssac (Tithonien inférieur, Quercy, Lot). Bulletin de la Société 868 herpétologique de France 96: 71-81.

869 Mazin JM, Hantzpergue P, Pouech J. 2016. The dinosaur tracksite of Loulle (early 870 Kimmeridgian; Jura, France). Geobios 49: 211-228.

871 Meyer CA. 1990. Sauropod tracks from the Upper Jurassic Reuchenette Formation 872 (Kimmeridgian; Lommiswil, Kt. Solothurn) of Northern Switzerland. Eclogae Geologicae 873 Helvetiae 83:389-397.

874 Meyer CA, Lockley MG.1997. Jurassic and Cretaceous dinosaur tracksites from Central Asia 875 (Uzbekistan and Turkmenistan). In: Yang, SY, Huh M, Lee YN, Lockley MG, eds. 876 Paleontological Society of Korea, Special Publication 2: 77-92.

877 Meyer CA, Thuring B. 2003. Dinosaurs of Switzerland. Comptes Rendus-Palevol. 2:103-117. 
878 Milàn J, Bromley RG. 2006. True tracks, undertracks and eroded tracks, experimental work with 879 tetrapod tracks in laboratory and field. Palaeogeography, Palaeoclimatology, Palaeoecology 880 231(3): 253-264.

881 Moreau JD, Gand G, Fara E, Michelin A. 2012. Biometric and morphometric approaches on 882 Lower Hettangian dinosaur footprints from the Rodez Strait (Aveyron, France).Comptes Rendus 883 Palevol 11(4): 231-239.

884 Moreau JD, Néraudeau D, Vullo R, Abit D, Mennecart B, Schnyder J.2017. Late Jurassic 885 dinosaur footprints from Chassiron-La Morelière (Oléron Island, western France). 886 Palaeobiodiversity and Palaeoenvironments 97(4):773-789.

887 Olsen PE. 1980. Fossil Great Lakes of the Newark Supergroup in New Jersey. In: Manspeizer 888 W. ed. Field Studies of New Jersey Geology and Guide to Field Trips 52nd Annual Meeting 889 New York State Geology Association, Newark College of Arts and Sciences, Newark, Rutgers 890 University, 352-398.

891 Olsen PE, Rainforth EC. 2003. The Early Jurassic ornithischian dinosaurian ichnogenus 892 Anomoepus. In: LeTourneau PM, Olsen PE, eds. The Great Rift Valleys of Pangea in Eastern 893 North America, Volume 2: Sedimentology, Stratigraphy, and Paleontology, Columbia University 894 Press, 314-367.

895 Olsen PE, Smith JB, McDonald NG.1998. Type material of the type species of the classic 896 theropod footprint genera Eubrontes, Anchisauripus, and Grallator (Early Jurassic, Hartford and 897 Deerfield basins, Connecticut and Massachusetts, USA). Journal of Vertebrate Paleontology 18: 898 586-601.

899 Pascual-Arribas C, Hernández-Medrano N. 2011. Posibles huellas de crías de terópodo en el 900 yacimiento de Valdehijuelos (Soria, España). Studia Geologica Salmanticensia 47 (1): 77-110.

901 Piñuela L. 2015. Huellas de dinosaurios y de otros reptiles del Jurásico Superior de Asturias. D. 902 Phil. Thesis, Oviedo University.

903 Razzolini NL, Vila B, Castanera D, Falkingham PL, Barco JL, Canudo JI, Manning PL, Galobart 904 À. (2014). Intra-trackway morphological variations due to substrate consistency: The El Frontal 905 dinosaur tracksite (Lower Cretaceous, Spain). PLOS ONE 9(4), e93708.

906 Razzolini NL, Belvedere M, Marty D, Paratte G, Lovis C, Cattin M, Meyer CA. 2017. 907 Megalosauripus transjuranicus ichnosp. nov. A new Late Jurassic theropod ichnotaxon from 908 NW Switzerland and implications for tridactyl dinosaur ichnology and ichnotaxomy. PLOS ONE 909 12(7): e0180289 
910 Santisteban C, Gaete R, Galobart A, Suñer M. 2003. Rastros de dinosaurios en el Jurásico 911 terminal (Facies Purbeck) de Corcolilla (Los Serranos, Valencia). In:Pérez Lorente F, Romero 912 Molina MM, Rivas Carrera, P, eds. Dinosaurios y otros reptiles mesozoicos en España. 33-40.

913 Santos VF. 2008. Pegadas de dinossáurios de Portugal. Museu Nacional de História Natural da 914 Universidade de Lisboa, Lisboa, 123 pp.

915 Schudack U, Schudack M, Marty D, Comment G. 2013. Kimmeridgian (Late Jurassic) ostracods 916 from Highway A16 (NW Switzerland): taxonomy, stratigraphy, palaeoecology, and 917 palaeobiogeography. Swiss Journal of Geosciences 106: 371-395.

918 Schulp AS, Al-Wosabi M. 2012. Telling apart ornithopod and theropod trackways: a closer look 919 at a large, Late Jurassic tridactyl dinosaur trackway at Serwah, Republic of Yemen. Ichnos 19(4): 920 194-198.

921 Stampfli G, Borel G. 2002. A plate tectonic model for the Paleozoic and Mesozoic constrained 922 by dynamic plate boundaries and restored synthetic oceanic isochrons. Earth and Planetary 923 Science Letters 196: 17-33.

924 Thierry J. 2000. Early Kimmeridgian. In: Dercourt J, Gaetani M, Vrielvynck B, Barrier E, Biju925 Duval B, Brunet MF, Cadet JP, Crasquin S, Sandulescu M, eds. Atlas Peri-Tethys, 926 Palaeogeographical maps - Explanatory Notes. CCGM/CGMN (Commission for the Geological 927 Map of the World), Paris: 85-97.

928 Thierry J, Barrier E, Abbate E, Alekseev AS, Ait-Salem H, Bouaziz S, et al. Map 10: Early 929 Kimmeridgian (146-144 Ma). 2000. In: Dercourt J, Gaetani M, Vrielvynck B, Barrier E, Biju930 Duval B, Brunet M, et al., editors. Atlas Peri-Tethys Palaeogeographical maps. CCGM/CGMN 931 (Commission for the Geological Map of the World), Paris.

932 Thulborn T. 1990. Dinosaur tracks. Chapman and Hall, London.

933 Valais SD.2011. Revision of dinosaur ichnotaxa from the La Matilde Formation (Middle 934 Jurassic), Santa Cruz Province, Argentina. Ameghiniana 48(1): 28-42.

935 .Waite R, Marty D, Strasser A, Wetzel A. 2013. The lost paleosols: Masked evidence for 936 emergence and soil formation on the Kimmeridgian Jura platform (NW Switzerland). 937 Palaeogeography, Palaeoclimatology, Palaeoecology 376: 73-90.

938 Weems RE. 1992. A re-evaluation of the taxonomy of Newark Supergroup saurischian dinosaur 939 tracks, using extensive statistical data from a recently exposed tracksite near Culpeper, Virginia. 940 In: Sweet PC, editor. Proceedings of the 26th forum on the geology of industrial minerals, 119: 941 113-127.

942 Wilson JA, Marsicano CA, Smith RM. 2009. Dynamic locomotor capabilities revealed by early 943 dinosaur trackmakers from Southern Africa. PLoS One, 4(10), e7331. 
944 Xing LD, Harris JD, Gierliński G. 2011. Therangospodus and Megalosauripus track assemblage 945 from the Upper Jurassic-Lower Cretaceous Tuchengzi Formation of Chicheng County, Hebei 946 Province, China and their paleoecological implications. Vertebrata PalAsiatica 49(4): 423-434.

947 Xing LD, Lockley MG, Klein H, Gierliński GD, Divay JD, Hu SM, Zhang JP, Ye Y, He YP. 948 2014. The non-avian theropod track Jialingpus from the Cretaceous of the Ordos Basin, China, 949 with a revision of the type material: implications for ichnotaxonomy and trackmaker 950 morphology. Palaeoworld 23:187-199.

951 Xing LD, Lockley MG, Hu N, Li G, Tong G, Matsukawa M, Klein H, Ye Y, Zhang J, Persons 952 WS. 2016. Saurischian track assemblages from the Lower Cretaceous Shenhuangshan Formation 953 in the Yuanma Basin, Southern China. Cretaceous Research 65: 1-9.

954

955

\section{FIGURE CAPTIONS:}

956

957

958

959

960

961

962

963

964

965

966

967

968

969

970

971

972

973

974

975

976

977

Fig. 1: Geographical and geological settings of the tracksites (modified from Razzolini et al., 2017; Marty et al., 2017). A) Geographical setting of the Ajoie district (NW Switzerland) with the location of the tracksites (1-Courtedoux-Béchat Bovais, 2- Courtedoux-Bois de Sylleux, 3- Courtedoux - Tchâfouè, 4- Courtedoux-Sur Combe Ronde, 5- Chevenez-Combe Ronde, 6Chevenez-La Combe) along Highway A16. B) Chrono-, bio- and lithostratigraphic setting of the Reuchenette Formation in the Ajoie district, Canton Jura, NW Switzerland (after Comment, Ayer \& Becker, 2011, 2015). Source credit: OCC-SAP, Canton Jura.

Fig. 2: Pictures and false-colour depth maps of the tracks with a high preservation grade that belong to the gracile morphotype. A) BEB500-T16-R3; B) BEB500-T26-R5; C) BEB500-T73L5; D) BSY1020-E2; E) CHV1000-E4; F) CRO500-T10-L10; G) SCR1055-T2-L2*; H) SCR1055-T3-L2*; I) TCH1055-E53; J) TCH1055-T2-L1; K) TCH1060-E58; L) TCH1065-E3; M) TCH1065-E177; N) TCH1065-T25-L2; O) TCH1069-T1-R2. *In these two cases, it is not a picture but a coloured mesh obtained from the $3 \mathrm{D}$-model. Scale bar $=5 \mathrm{~cm}$. Source credit: OCCSAP, Canton Jura.

Fig. 3: Pictures and false-colour depth maps of the tracks with a high preservation grade that belong to the robust morphotype. A) BEB500-T120-R5; B) BEB500-T120-R6; C) TCH1065T21-R1; D) TCH1065-E188; E) TCH1065-E124; F) TCH1065-T15-R1. Scale bar = $5 \mathrm{~cm}$. Source credit: OCC-SAP, Canton Jura.

Fig. 4: Morphological variation in the footprint shape along the studied trackways from BEB500 tracksite. A) BEB500-T16 (gracile morphotype); B) BEB500-T17 (gracile morphotype); C) BEB500-T58 (gracile morphotype); D) BEB500-T73 (gracile morphotype); E) BEB500-T75 (gracile morphotype); F) BEB500-T78 (gracile morphotype); G) BEB500-T82 (gracile 
978

979

980

981

982

983

984

985

986

987

988

989

990

991

992

993

994

995

996

997

998

999

1000

1001

1002

1003

1004

1005

1006

1007

1008

1009

1010

1011

1012

1013

1014

1015

morphotype); H) BEB500-T120 (robust morphotype). I) BEB500-T93 (gracile morphotype). Source credit: OCC-SAP, Canton Jura.

Fig. 5: Morphological variation in the footprint shape along the studied trackways from the CRO500, TCH1055, TCH1065 and TCH1069 tracksites. A) CRO500-T10 (gracile morphotype); B) CRO500-T30BIS (gracile morphotype); C) TCH1055-T2 (gracile morphotype); D) TCH1065-T15 (robust morphotype); E) TCH1069-T2 (robust morphotype); F) TCH1065-T25 (gracile morphotype). Source credit: OCC-SAP, Canton Jura.

Fig. 6: Bivariate graph plotting the footprint length/footprint width ratio against the mesaxony (AT) of the studied tracks (gracile and robust morphotype) with the larger tracks described in the Reuchenette Formation. A) Gracile and robust morphotype compared with Megalosauripus tracks (including tracks classified as Megalosauripus transjuranicus, Megalosauripus cf. transjuranicus and Megalosauripus isp.), the Morphotype II tracks and Jurabrontes curtedulensis (after Razzolini et al., 2017; Marty et al., 2017). Note that in many cases the points represent tracks from the same trackway, so variation through the trackway is also represented. B) The studied tracks compared with just the holotype and paratype specimens of Megalosauripus transjuranicus and Jurabrontes curtedulensis, plus the best-preserved tracks of Morphotype II (BEB500-TR7). Outline drawings not to scale. The specimen in red is CRO500T10-L10 (previously classified as Carmelopodus and herein consider as part of the gracile morphotype, see discussion). Source credit: OCC-SAP, Canton Jura.

Fig. 7: Main small-medium-sized tridactyl dinosaur footprints described in the Late Jurassic of Europe. A) Grallator from Spain (S, after Castanera, Piñuela \& García-Ramos, 2016); B) Anomoepus from Spain (S, after Piñuela, 2015); C) Carmelopodus from France (C, after Mazin, Hantzpergue \& Pouech, 2016); D) Eubrontes from France (C, after Mazin et al., 2000); E) Wildeichnus from Poland (C, after Gierliński, Niedźwiedzki \& Nowacki, 2009); F) Jialingpus from Poland (C, after Gierliński, Niedźwiedzki \& Nowacki, 2009). G) Dineichnus from Poland (C, after Gierliński, Niedźwiedzki \& Nowacki, 2009); H) Dineichnus from Portugal (S, Lockley et al., 1998a); I) Therangospodus-like track from Portugal (S, after Lockley, Meyer \& Moratalla, 2000; J) Grallator from Germany (S, after Diedrich, 2011). K) Therangospodus-like track from Italy (C, after Conti et al., 2005); L) Carmelopodus-like track from Italy (C, after Conti et al., 2005). Scale bar $=1 \mathrm{~cm}(\mathrm{E}), 5 \mathrm{~cm}(\mathrm{~A}, \mathrm{~F}, \mathrm{G}), 10 \mathrm{~cm}(\mathrm{~B}, \mathrm{C}, \mathrm{D}, \mathrm{H}, \mathrm{I}, \mathrm{J}, \mathrm{K}, \mathrm{L}) . \mathrm{S}$ and $\mathrm{C}$ refer to siliciclastic and carbonate substrate, respectively. Source credit: OCC-SAP, Canton Jura.

Fig. 8: A) Outline drawing of the holotype of Carmelopodus untermannorum (S, redrawn after Lockley et al., 1998b); B) Outline drawing of the holotype of Wildeichnus navesi (V, redrawn after Lockley, Mitchel \& Odier, 2007); C) Outline drawing of the topotype of Therangospodus pandemicus (S, after Lockley, Meyer \& Moratalla, 2000); D) Outline drawing of Anomoepus scambus (S, after Olsen \& Rainforth, 2003); E) Outline drawing of the holotype of Dineichnus socialis (S, after Lockley et al., 1998a); F) Composite outline drawing of type trackway of Grallator parallelus (S, redrawn from Olsen, Smith \& McDonald, 1998); G) Outline drawing of 
1016 type specimen of Anchisauripus sillimani (S, redrawn from Olsen, Smith \& McDonald, 1998); $1017 \mathrm{H}$ ) Outline drawing of type specimen of Eubrontes giganteus (S, redrawn from Olsen, Smith \& 1018 McDonald, 1998). I) Outline drawing of type specimen of Jialingpus yuechiensis (S, redrawn 1019 from Lockley et al., 2013); J) Outline drawing of type specimen of Kalohipus bretunensis (S, 1020 redrawn from Fuentes Vidarte \& Meijide Calvo, 1998). K) Outline drawing of type specimen of 1021 Jurabrontes curtedulensis (redrawn from Marty et al., 2017). L) Outline drawing of type 1022 specimen of Megalosauripus transjuranicus (redrawn from Razzolini et al., 2017). M) Outline 1023 drawing of specimen BSY1020-E2 (cf. Kalohipus isp.). N) Outline drawing of specimen 1024 CRO500-T10-L10 (cf. Kalohipus isp.). O) Outline drawing of specimen TCH-1060-E58 (cf. 1025 Kalohipus isp.); P) Outline drawing of specimen TCH-1065-T21-R1 (cf. Therangospodus isp.). 1026 Q) Outline drawing of specimen BEB500-T120-R5 (Therangospodus? isp.). S, C and V refer to 1027 siliciclastic, carbonate and volcanoclastic substrate, respectively. Scale bar $=2 \mathrm{~cm}(\mathrm{~B}, \mathrm{D}), 5 \mathrm{~cm}$ 1028 (F,G, H, I, J), 10 cm (A, C, E, L, M-Q), 50 cm (K). Source credit: OCC-SAP, Canton Jura.

1029

1030

1031

1032

1033

1034

1035

1036

1037

1038

1039

1040

1041

1042

1043

1044

1045

1046

1047

1048

1049

1050

1051

1052

1053

1054
Fig. 9: Bivariate graph plotting the footprint length/footprint width ratio vs AT of the studied tracks (gracile and robust morphotype) with some of the main dinosaur tridactyl ichnotaxa mentioned in the text. Outline drawings not to scale. Source credit: OCC-SAP, Canton Jura.

Table 1: Measurements of the specimens with a high preservation grade: footprint length (FL), footprint width (FW), footprint length /footprint width ratio (FL/FW), digit length (LI, LII, LIII), digit width (WI, WII, WIII), divarication angles (II-III, III-IV), mesaxony (AT, anterior triangle ratio; ATw, anterior triangle width; ATl, anterior triangle length).

Table 2: Variation in preservation grade and variation in maximum depth (difference between highest and lowest value) through the analysed trackways (see Table S1 for specific values of each track).

Supplemental information Table S1: List of the specimens analysed, their quality of preservation (preservation grade) and the maximum depth. Those with preservation grade $0-0.5$ are not included in the figshare file. The tracks where the variation along the trackway has been analysed are in red.

Supplemental information Figure S1: Map of the Courtedoux-Béchat Bovais tracksite, level 500 (BEB500). In red (gracile) and blue (robust) the minute to medium-sized tridactyl tracks and in green, the larger morphtoype (Morphotype II). Source credit: OCC-SAP, Canton Jura.

Supplemental information Figure S2: Map of the Courtedoux-Tchâfouè tracksite, level 1065 (TCH1065). In red (gracile) and blue (robust) the minute to medium-sized tridactyl tracks and in green, the larger morphtoype (Jurabrontes curtedulensis see Marty et al., 2017). Source credit: OCC-SAP, Canton Jura.

Supplemental information Figure S3: Map of the Chevenez-Combe Ronde, level 500 (CRO500). In red (gracile) and blue (robust) the minute to medium-sized tridactyl tracks and in green, the larger morphtoype (Morphotype II). Source credit: OCC-SAP, Canton Jura. 
1057 We thank all technicians, photographers, geometers, drawers, collection managers, and 1058 preparators of the PALA16 that were involved during the excavation and documentation of the 1059 tracksites and during the set-up and organization of the track collection. We also thank the 1060 scientific staff of the PALA16 and JURASSICA Muséum for various stimulating discussions and 1061 valuable input. The authors also thank Laura Piñuela, Vanda Santos, Ignacio Díaz-Martínez, 1062 Oliver W.M. Rauhut and Novella L. Razzolini for fruitful discussion on the topic of this 1063 manuscript. The comments of the editor (Claudia Marsicano) and the reviewers (Jesper Milàn 1064 and Verónica Krapovickas) have considerably improved the quality of the manuscript and are 1065 greatly appreciated. Rupert Glasgow revised the English grammar. 
Figure 1

Geographical and geological settings of the Highway 16 tracksites (modified from Razzolini et al., 2017; Marty et al., 2017).

A) Geographical setting of the Ajoie district (NW Switzerland) with the location of the tracksites (1- Courtedoux-Béchat Bovais, 2- Courtedoux-Bois de Sylleux, 3-

Courtedoux-Tchâfouè, 4- Courtedoux-Sur Combe Ronde, 5- Chevenez-Combe Ronde, 6Chevenez-La Combe) along Highway A16. B) Chrono-, bio- and lithostratigraphic setting of the Reuchenette Formation in the Ajoie district, Canton Jura, NW Switzerland (after Comment, Ayer \& Becker, 2011, 2015). Source credit: OCC-SAP, Canton Jura.
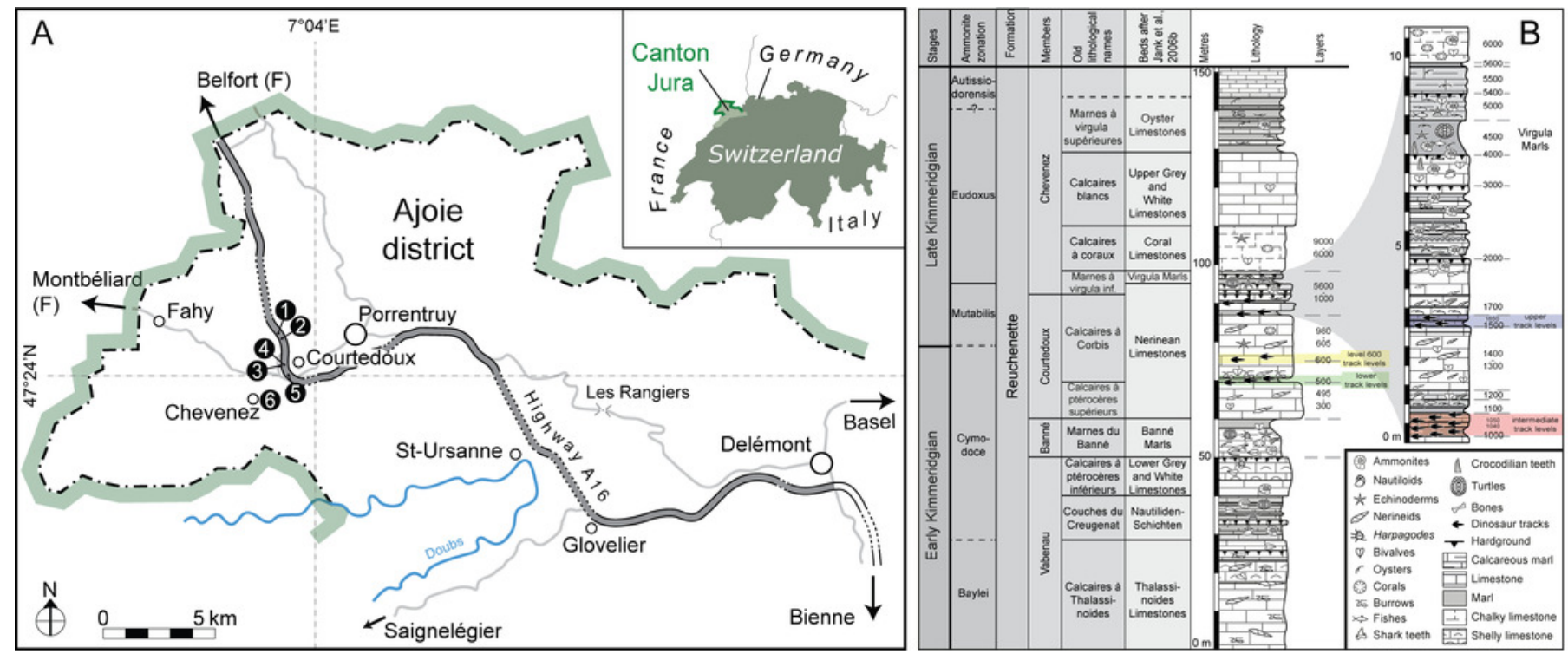


\section{Figure 2}

Pictures and false-colour depth maps of the tracks with a high preservation grade that belong to the gracile morphotype.

A) BEB500-T16-R3; B) BEB500-T26-R5; C) BEB500-T73-L5; D) BSY1020-E2; E) CHV1000-E4; F) CRO500-T10-L10; G) SCR1055-T2-L2*; H) SCR1055-T3-L2*; I) TCH1055-E53; J) TCH1055-T2L1; K) TCH1060-E58; L) TCH1065-E3; M) TCH1065-E177; N) TCH1065-T25-L2; O) TCH1069T1-R2. *In these two cases, it is not a picture but a coloured mesh obtained from the 3Dmodel. Scale bar $=5 \mathrm{~cm}$. Source credit: OCC-SAP, Canton Jura. 

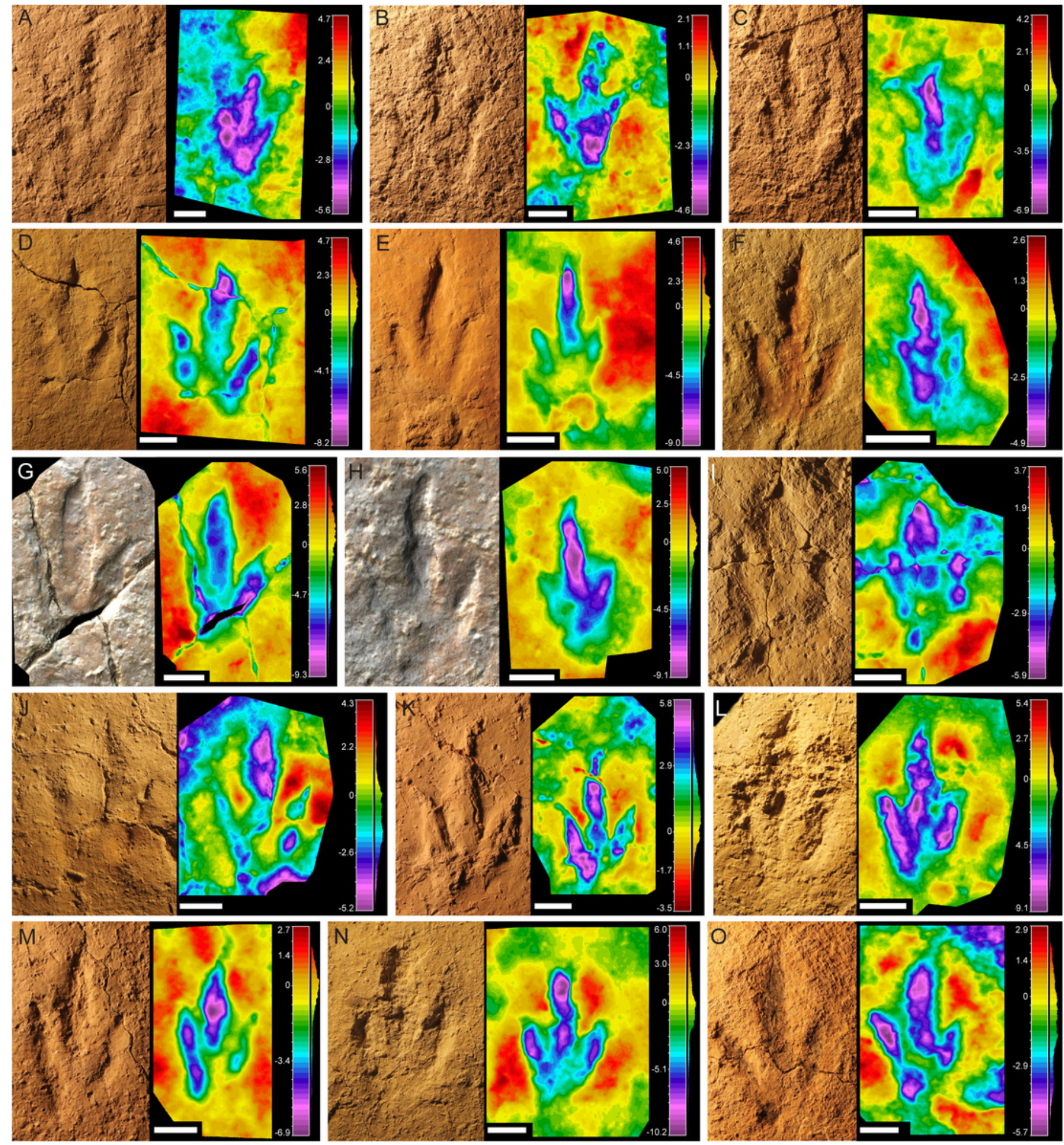
Figure 3

Pictures and false-colour depth maps of the tracks with a high preservation grade that belong to the robust morphotype.

A) BEB500-T120-R5; B) BEB500-T120-R6; C) TCH1065-T21-R1; D) TCH1065-E188; E)

TCH1065-E124; F) TCH1065-T15-R1. Scale bar $=5 \mathrm{~cm}$. Source credit: OCC-SAP, Canton Jura.
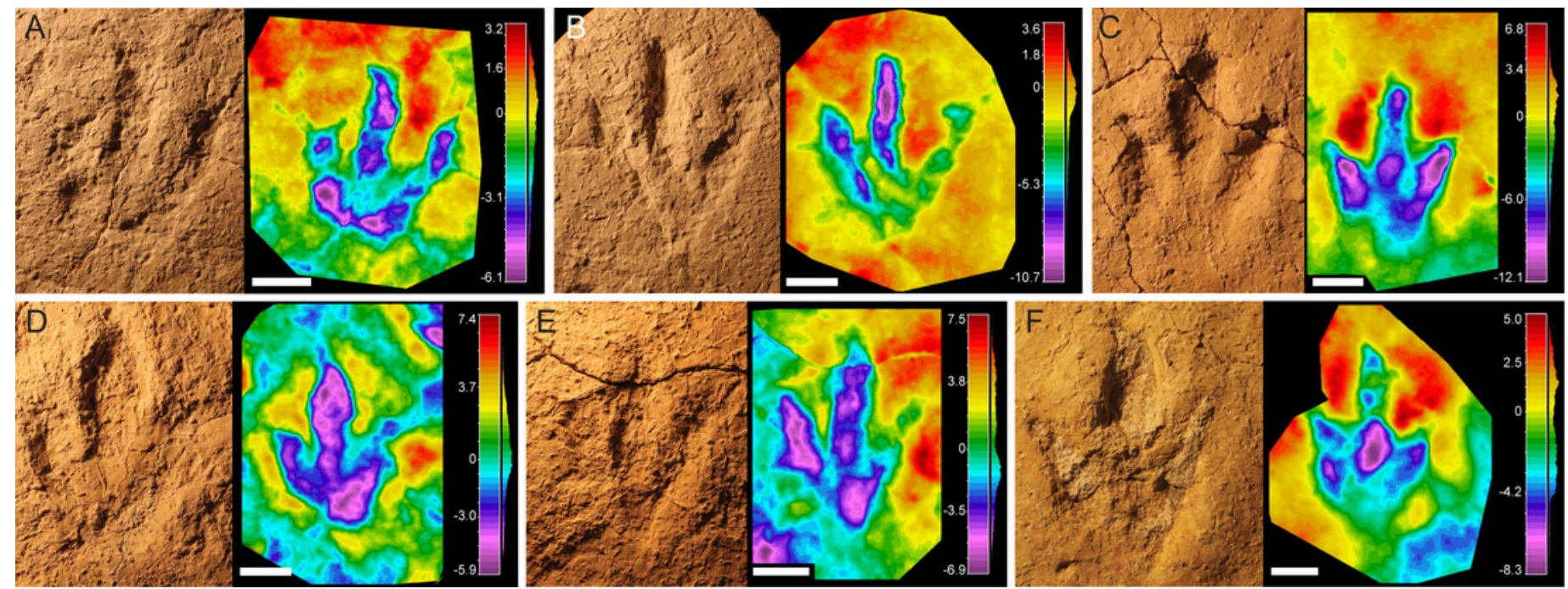


\section{Figure 4}

Morphological variation in the footprint shape along the studied trackways from BEB500 tracksite.
A) BEB500-T16 (gracile morphotype); B) BEB500-T17 (gracile morphotype); C) BEB500-T58 (gracile morphotype); D) BEB500-T73 (gracile morphotype); E) BEB500-T75 (gracile morphotype); F) BEB500-T78 (gracile morphotype); G) BEB500-T82 (gracile morphotype); H) BEB500-T120 (robust morphotype). I) BEB500-T93 (gracile morphotype). Source credit: OCC- SAP, Canton Jura. 
A

14 )

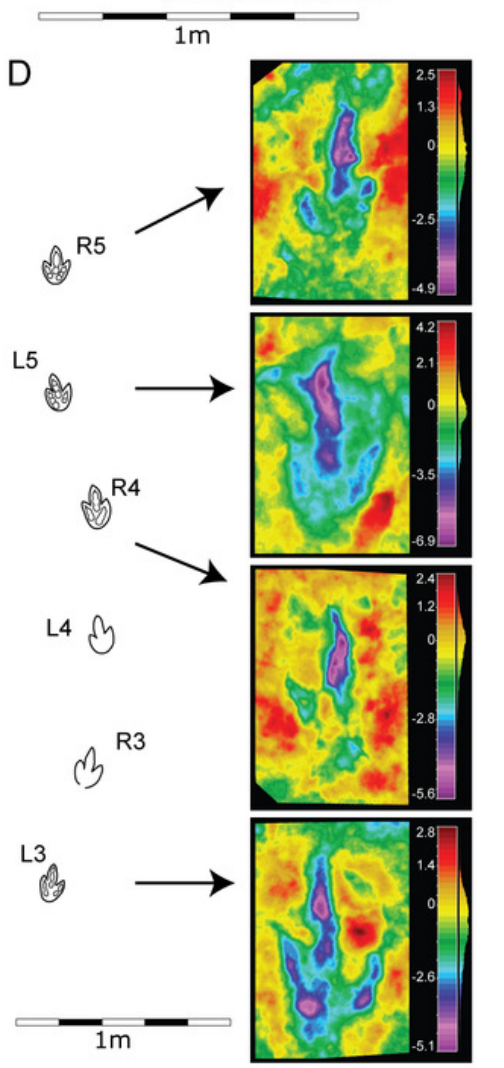

G

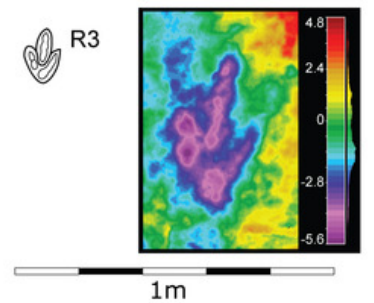

D
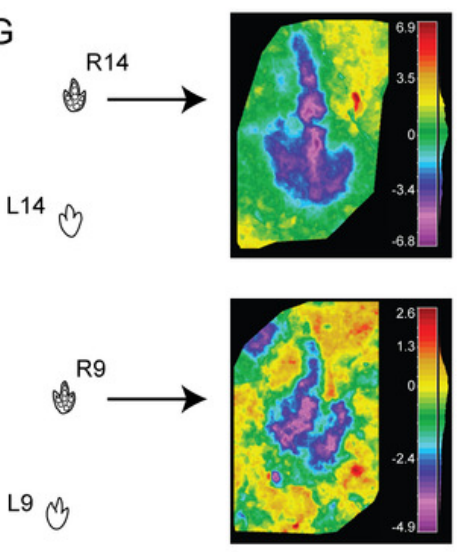

L8 $\mathrm{OH} \longrightarrow$

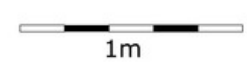

E

L15 B
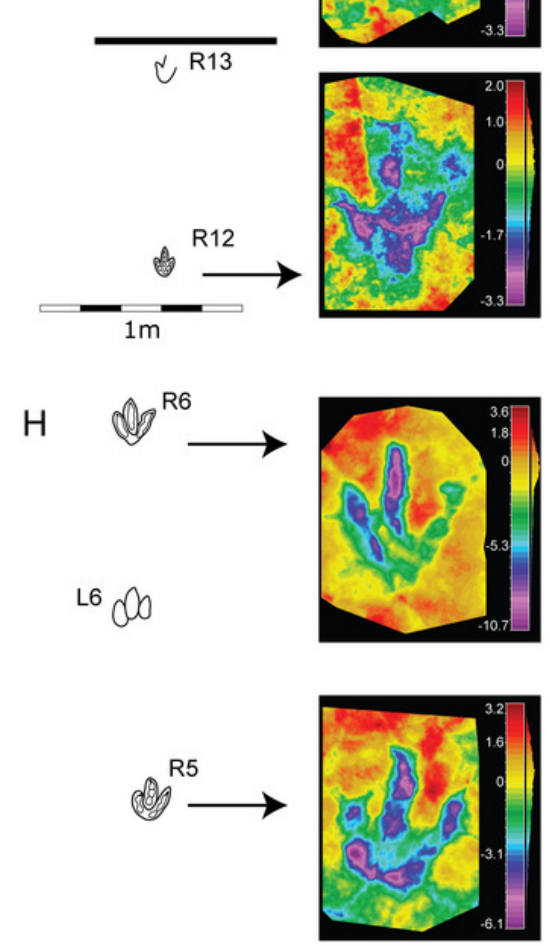

L5 강 $\longrightarrow$

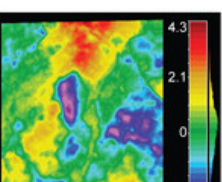

R4

:NEW 2 Mar 2018)

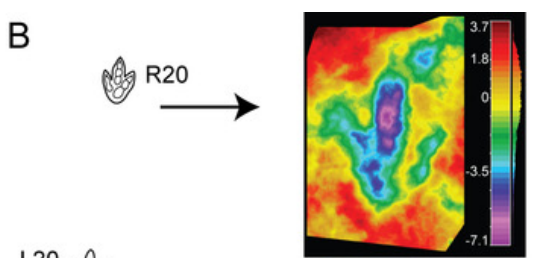

C

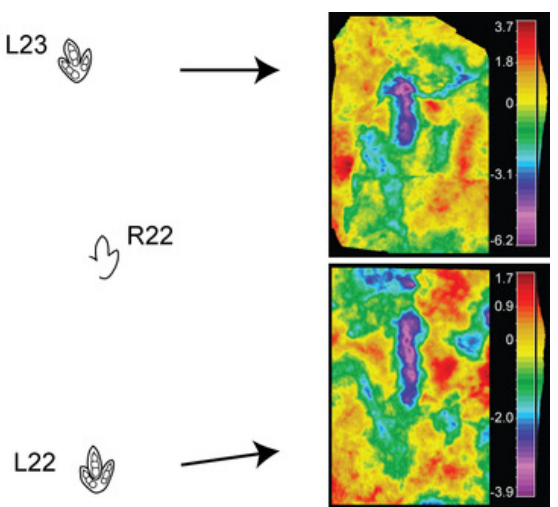

$\mathrm{L} 22$ (8) $\longrightarrow$.

21

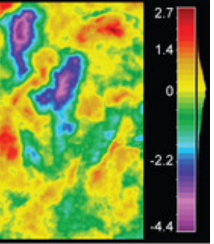

${ }^{\mathrm{R}}{ }^{\mathrm{R} 21} \longrightarrow$
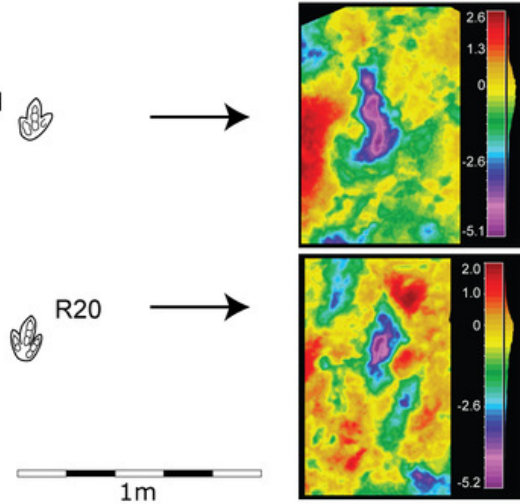

F L5
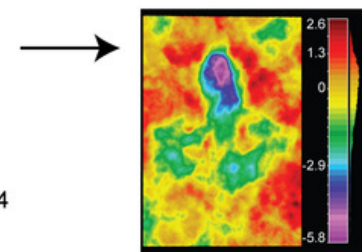

B R4

L4
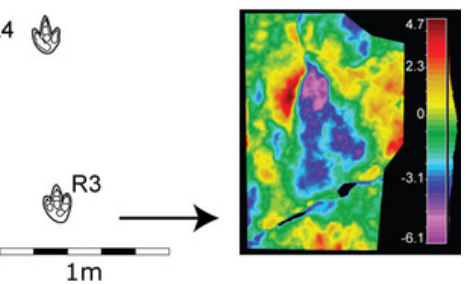

I
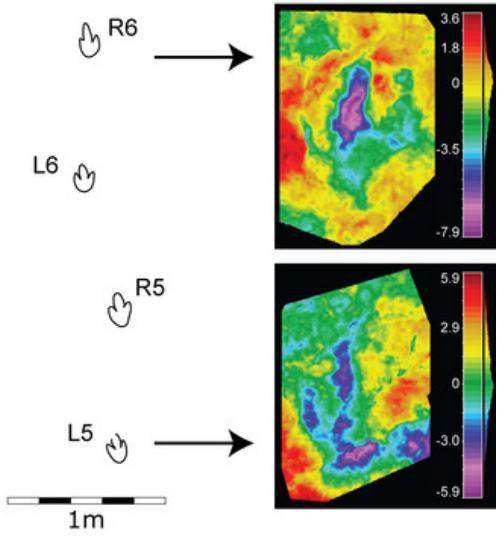


\section{Figure 5}

Morphological variation in the footprint shape along the studied trackways from the CRO500, TCH1055, TCH1065 and TCH1069 tracksites.

A) CRO500-T10 (gracile morphotype); B) CRO500-T30BIS (gracile morphotype); C) TCH1055-

T2 (gracile morphotype); D) TCH1065-T15 (robust morphotype); E) TCH1069-T2 (robust

morphotype); F) TCH1065-T25 (gracile morphotype). Source credit: OCC-SAP, Canton Jura. 
A
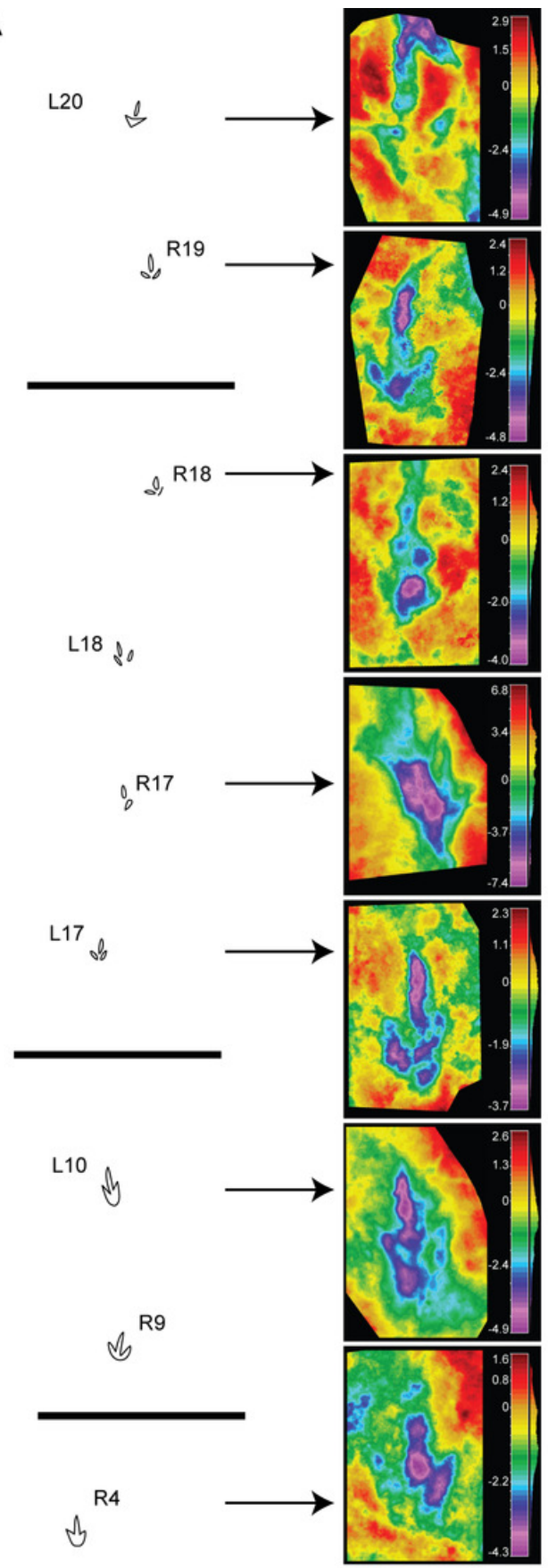

${ }^{\mathrm{L}} \mathrm{b}$

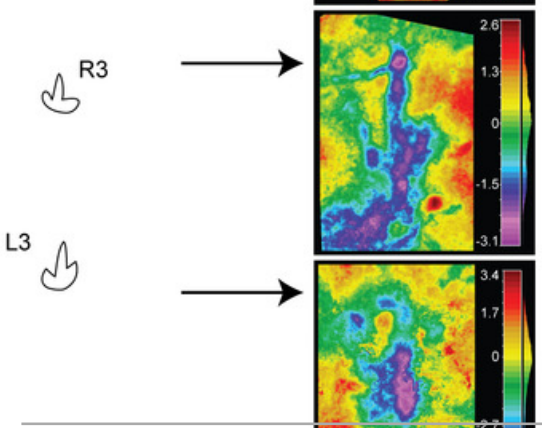

B
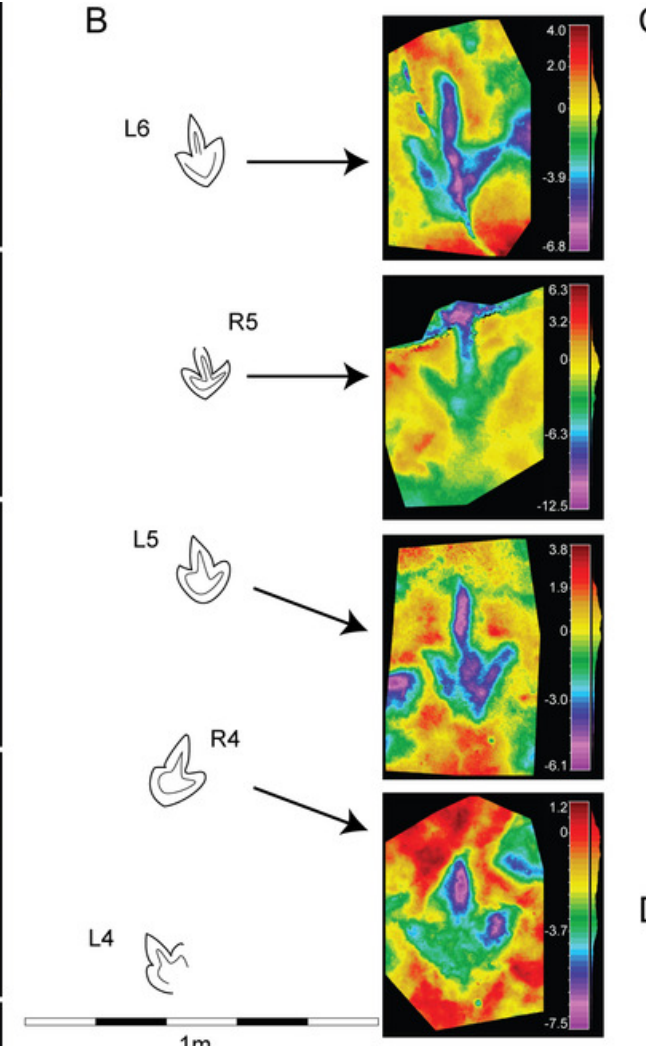

E

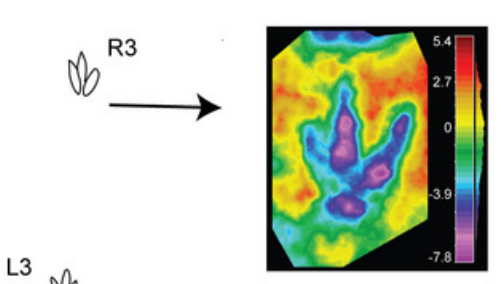

L3 8

$\mathrm{F}$
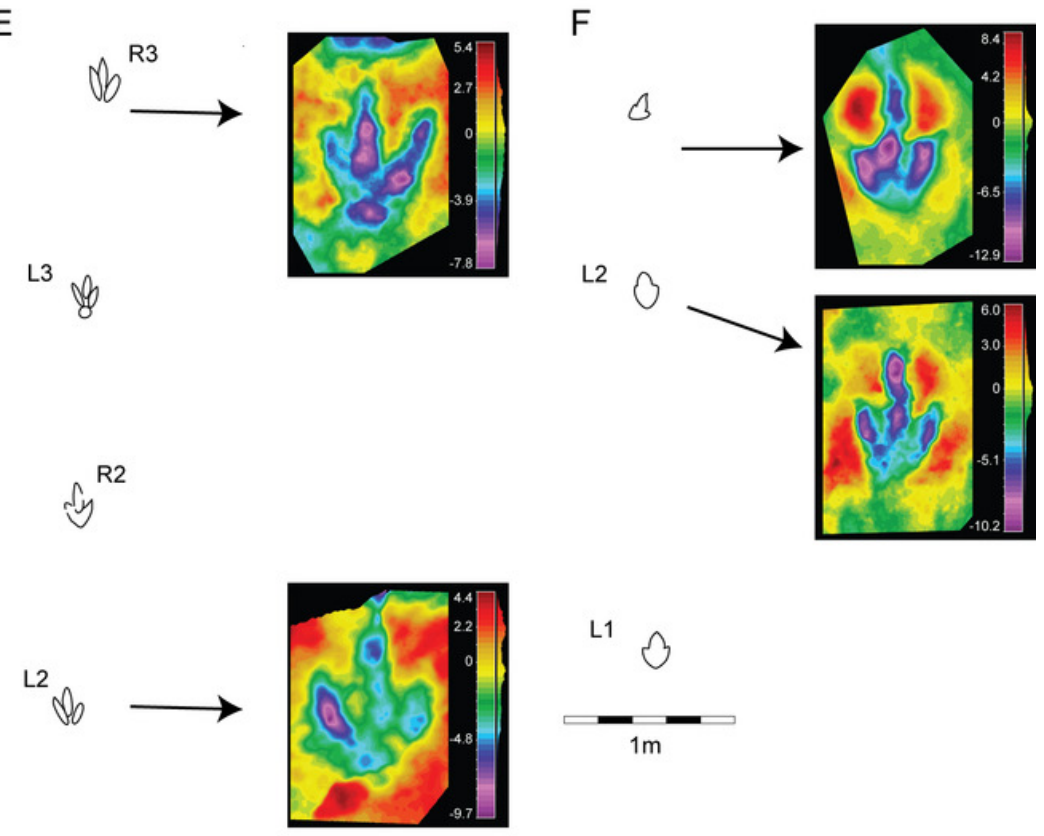

Di

C

$\omega^{R 2}$
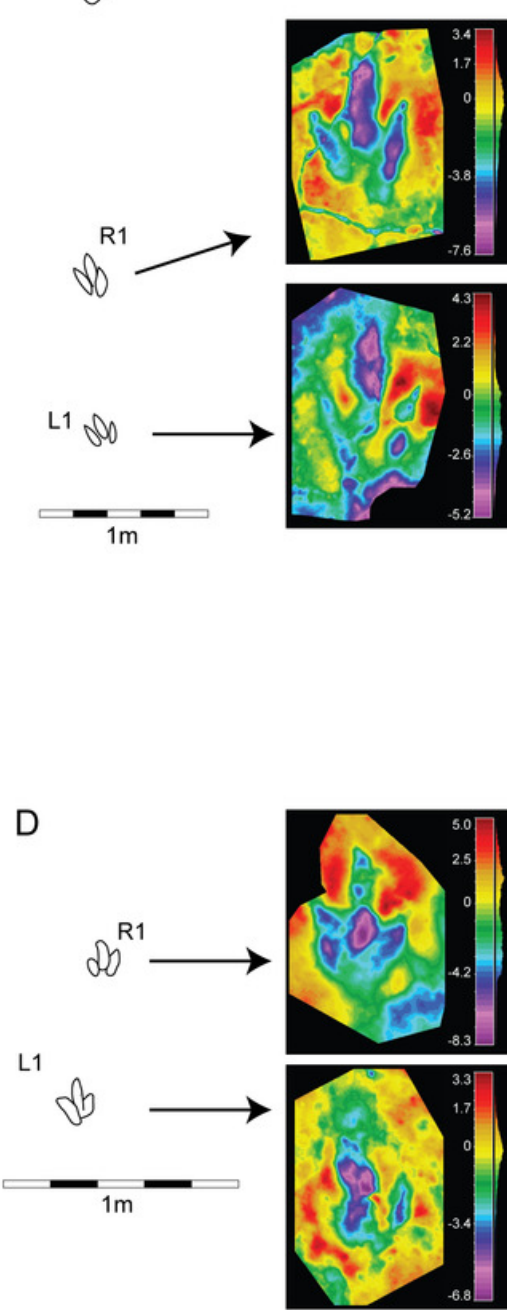

$\sqrt{y}^{\mathrm{R} 2}$

L1 0

$-$ 


\section{Figure 6}

Bivariate graph plotting the footprint length/footprint width ratio against the mesaxony (AT) of the studied tracks (gracile and robust morphotype) with the larger tracks described in the Reuchenette Formation.

A) Gracile and robust morphotype compared with Megalosauripus tracks (including tracks classified as Megalosauripus transjuranicus, Megalosauripus cf. transjuranicus and Megalosauripus isp.), the Morphotype II tracks and Jurabrontes curtedulensis (after Razzolini et al., 2017; Marty et al., 2017). Note that in many cases the points represent tracks from the same trackway, so variation through the trackway is also represented. B) The studied tracks compared with just the holotype and paratype specimens of Megalosauripus transjuranicus and Jurabrontes curtedulensis, plus the best-preserved tracks of Morphotype II (BEB500-TR7). Outline drawings not to scale. The specimen in red is CRO500-T10-L10 (previously classified as Carmelopodus and herein consider as part of the gracile morphotype, see discussion). Source credit: OCC-SAP, Canton Jura. 

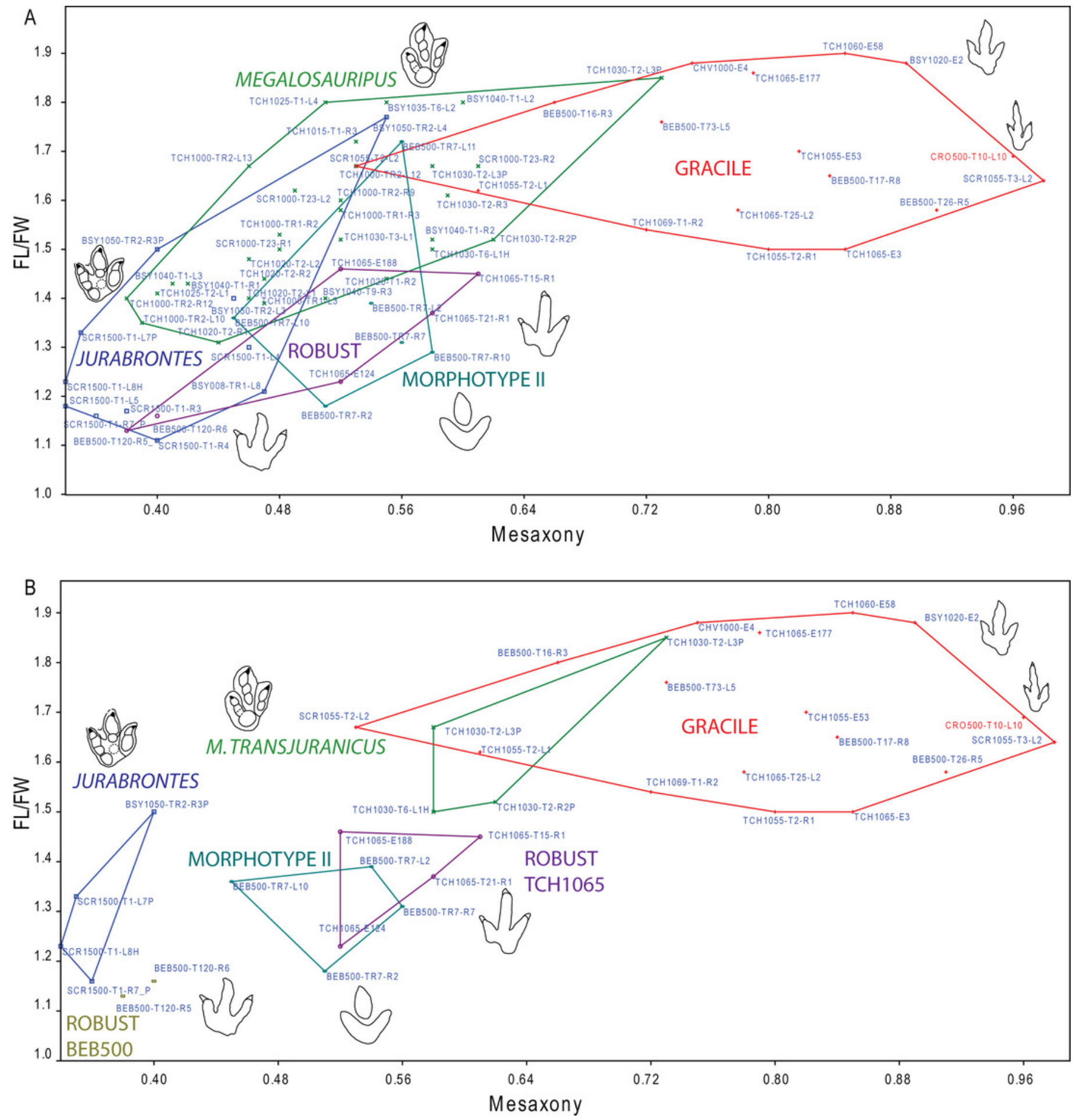


\section{Figure 7}

Main small-medium-sized tridactyl dinosaur footprints described in the Late Jurassic of Europe.

A) Grallator from Spain (S, after Castanera, Piñuela \& García-Ramos, 2016); B) Anomoepus from Spain (S, after Piñuela, 2015); C) Carmelopodus from France (C, after Mazin, Hantzpergue \& Pouech, 2016); D) Eubrontes from France (C, after Mazin et al., 2000); E) Wildeichnus from Poland (C, after Gierliński, Niedźwiedzki \& Nowacki, 2009); F) Jialingpus from Poland (C, after Gierliński, Niedźwiedzki \& Nowacki, 2009). G) Dineichnus from Poland (C, after Gierliński, Niedźwiedzki \& Nowacki, 2009); H) Dineichnus from Portugal (S, Lockley et al., 1998a); I) Therangospodus-like track from Portugal (S, after Lockley, Meyer \& Moratalla, 2000; J) Therangospodus-like track from Italy (C, after Conti et al., 2005). K) Carmelopodus-like track from Italy (C, after Conti et al., 2005); L) Grallator from Germany (S, after Diedrich, 2011). Scale bar $=1 \mathrm{~cm}(E), 5 \mathrm{~cm}(A, F, G), 10 \mathrm{~cm}(B, C, D, H, I, J, K, L) . S$ and C refer to siliciclastic and carbonate substrate, respectively. Source credit: OCC-SAP, Canton Jura.

A

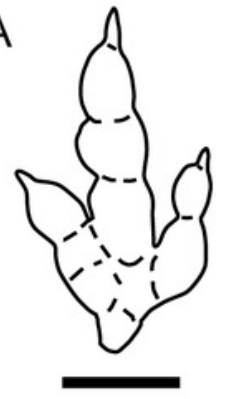

G

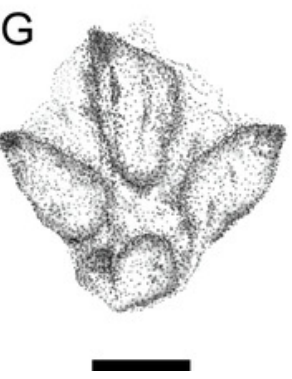

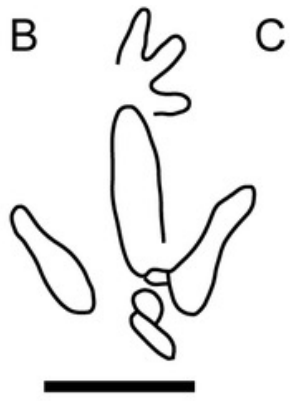
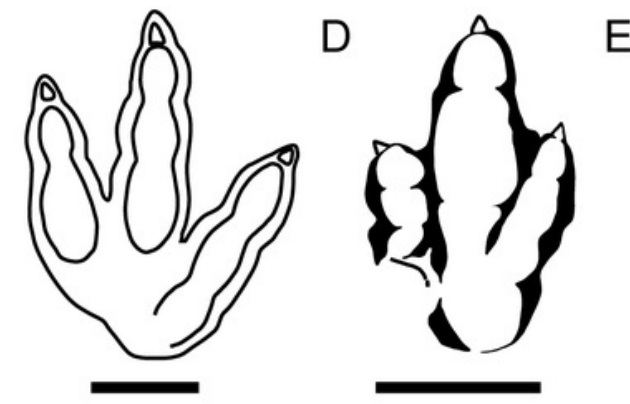

$\mathrm{E}$

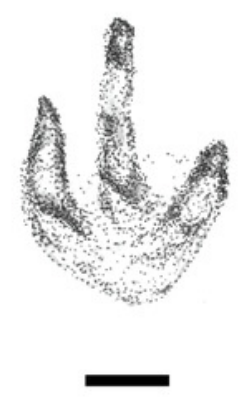

$\mathrm{F}$
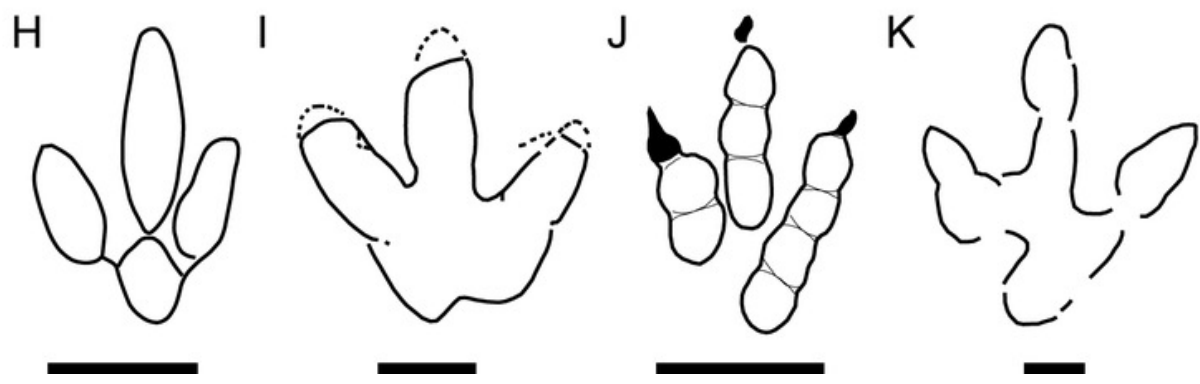

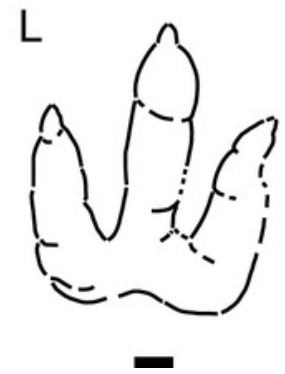




\section{Figure 8}

Small-medium-sized tridactyl dinosaur ichnotaxa with affinities with the described morphotypes.

A) Outline drawing of the holotype of Carmelopodus untermannorum (S, redrawn after Lockley et al., 1998b); B) Outline drawing of the holotype of Wildeichnus navesi (V, redrawn after Lockley, Mitchel \& Odier, 2007); C) Outline drawing of the topotype of Therangospodus pandemicus (S, after Lockley, Meyer \& Moratalla, 2000); D) Outline drawing of Anomoepus scambus (S, after Olsen \& Rainforth, 2003); E) Outline drawing of the holotype of Dineichnus socialis (S, after Lockley et al., 1998a); F) Composite outline drawing of type trackway of Grallator parallelus (S, redrawn from Olsen, Smith \& McDonald, 1998); G) Outline drawing of type specimen of Anchisauripus sillimani (S, redrawn from Olsen, Smith \& McDonald, 1998); H) Outline drawing of type specimen of Eubrontes giganteus (S, redrawn from Olsen, Smith \& McDonald, 1998). I) Outline drawing of type specimen of Jialingpus yuechiensis (S, redrawn from Lockley et al., 2013); J) Outline drawing of type specimen of Kalohipus bretunensis (S, redrawn from Fuentes Vidarte \& Meijide Calvo, 1998). K) Outline drawing of type specimen of Jurabrontes curtedulensis (redrawn from Marty et al., 2017). L) Outline drawing of type specimen of Megalosauripus transjuranicus (redrawn from Razzolini et al., 2017). M) Outline drawing of specimen BSY1020-E2 (cf. Kalohipus isp.). N) Outline drawing of specimen CRO500-T10-L10 (cf. Kalohipus isp.). O) Outline drawing of specimen TCH-1060-E58 (cf. Kalohipus isp.); P) Outline drawing of specimen TCH-1065-T21-R1 (cf. Therangospodus isp.). Q) Outline drawing of specimen BEB500-T120-R5 (Therangospodus? isp.). S, C and V refer to siliciclastic, carbonate and volcanoclastic substrate, respectively. Scale bar $=2 \mathrm{~cm}(B, D), 5$ $\mathrm{cm}(\mathrm{F}, \mathrm{G}, \mathrm{H}, \mathrm{I}, \mathrm{J}), 10 \mathrm{~cm}(\mathrm{~A}, \mathrm{C}, \mathrm{E}, \mathrm{L}, \mathrm{M}-\mathrm{Q}), 50 \mathrm{~cm}(\mathrm{~K})$. Source credit: OCC-SAP, Canton Jura. 

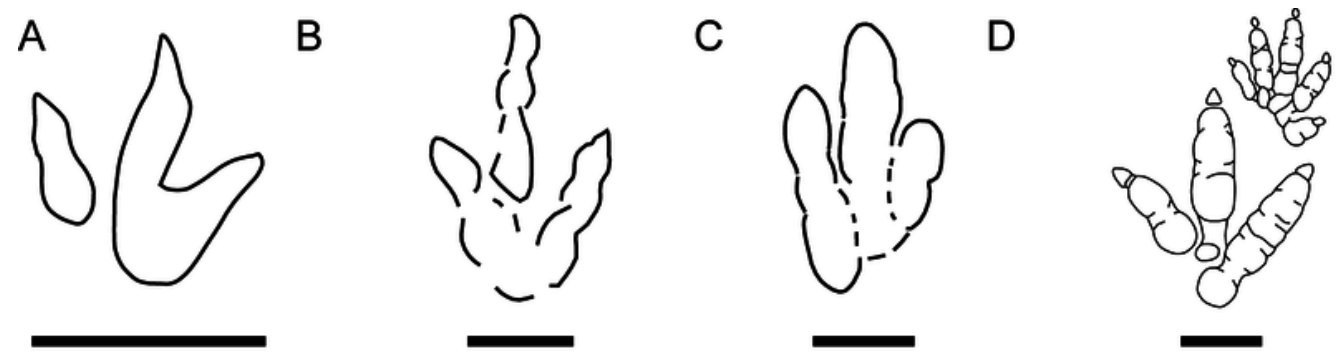

E

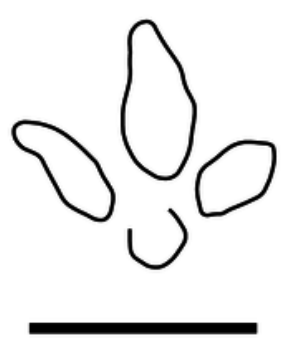

$\mathrm{F}$
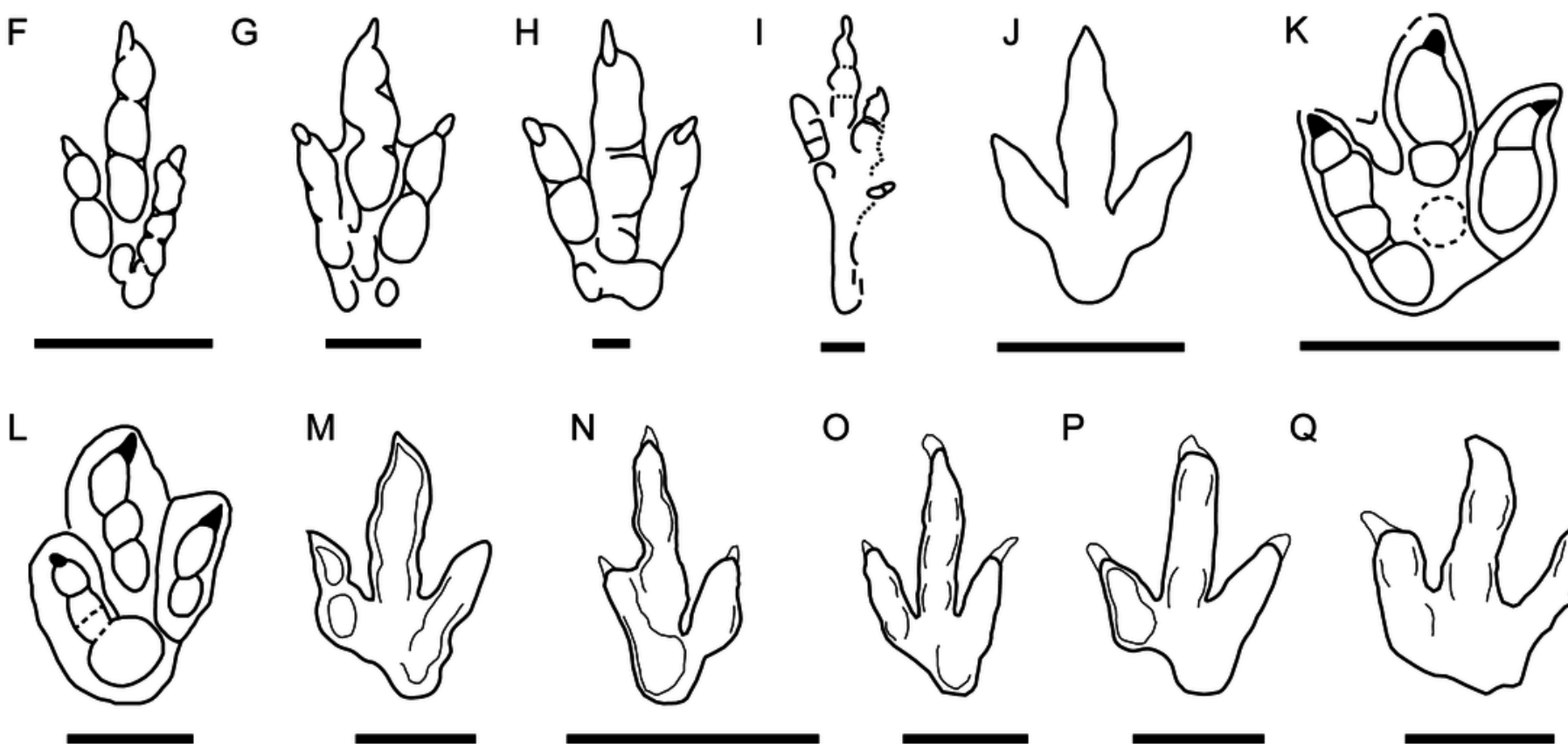

Q

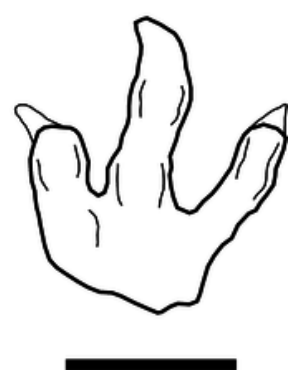


Figure 9

Bivariate graph plotting the footprint length/footprint width ratio against AT of the studied tracks (gracile and robust morphotype) with some of the main dinosaur tridactyl of ichnotaxa mentioned in the text.

Outline drawings not to scale. Source credit: OCC-SAP, Canton Jura.

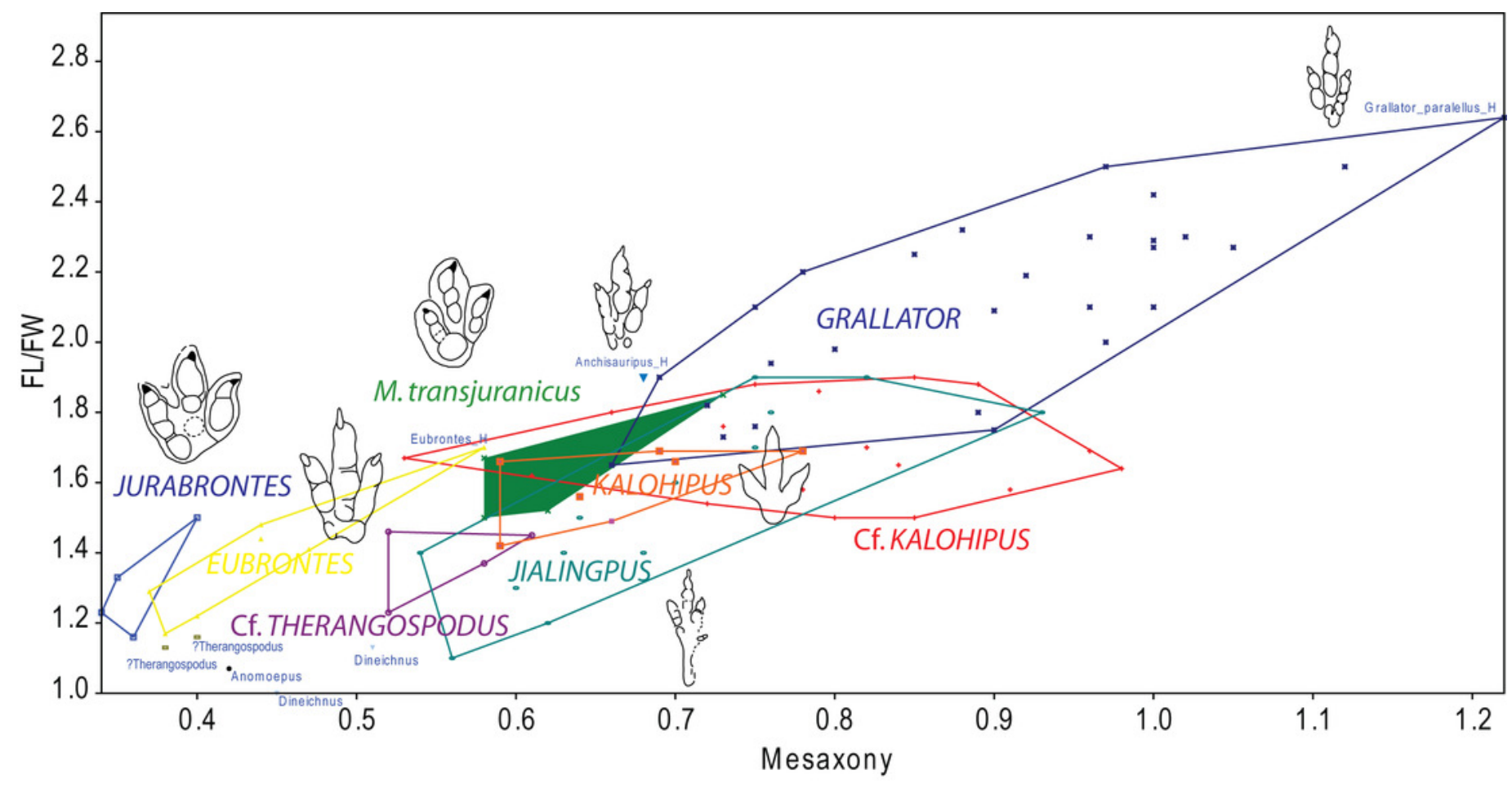




\section{Table $\mathbf{1}$ (on next page)}

Measurements of the specimens with a high preservation grade:

footprint length (FL), footprint width (FW), footprint length /footprint width ratio (FL/FW), digit length (LI, LII, LIII), digit width (WI, WII, WIII), divarication angles (II-III, III-IV), mesaxony (AT, anterior triangle ratio; ATw, anterior triangle width; ATI, anterior triangle length). 


\begin{tabular}{|c|c|c|c|c|c|c|c|c|c|c|c|c|c|c|}
\hline Track & $\mathrm{FL}$ & FW & FL/FW & LII & LIII & LIV & WII & WIII & WIV & $\left\|{ }^{\wedge}\right\| \|$ & $\mathrm{III} I^{\wedge} \mathrm{IV}$ & ATw & Atl & AT \\
\hline BEB500-T16-R3 & 18 & 10 & 1.8 & 13.5 & 18 & 13.8 & 2 & 1.9 & 1.8 & 22.5 & 17.5 & 8.8 & 5.8 & 0.66 \\
\hline BEB500-T17-R8 & 19 & 11.5 & 1.65 & 11 & 19 & 13 & 1.9 & 3.3 & 1.6 & 23 & 20 & 10.5 & 8.8 & 0.84 \\
\hline BEB500-T26-R5 & 19 & 12 & 1.58 & 13 & 19 & 14 & 2.2 & 3 & 2.9 & 32 & 26 & 10.3 & 9.4 & 0.91 \\
\hline BEB500-T73-L5 & 15 & 8.5 & 1.76 & 8.5 & 15 & 10 & 2.3 & 2.9 & 2.5 & 31 & 22 & 7.9 & 5.8 & 0.73 \\
\hline BSY1020-E2 & 22 & 11.7 & 1.88 & 15 & 22 & 13.5 & 3.6 & 3 & 2.7 & 21.5 & 24.5 & 9.5 & 8.5 & 0.89 \\
\hline TCH1055-E53 & 17.5 & 10.3 & 1.7 & 12.2 & 17.5 & 12 & 3 & 2.7 & 2.5 & 25 & 17.5 & 8.5 & 7 & 0.82 \\
\hline TCH1055-T2-L1 & 21.2 & 13.1 & 1.62 & 15.6 & 21.2 & 15 & 2.3 & 2.1 & 2.2 & 25 & 22 & 11.4 & 7 & 0.61 \\
\hline TCH1055-T2-R1 & 19.5 & 13 & 1.5 & 13.2 & 20.5 & 13.1 & 3.3 & 3.7 & 2.5 & 29 & 23 & 10.6 & 8.5 & 0.80 \\
\hline TCH1060-E58 & 20 & 10.5 & 1.90 & 20 & 13.5 & 12 & 3.4 & 3.1 & 2.9 & 27 & 22 & 8.8 & 7.5 & 0.85 \\
\hline TCH1065-E177 & 17.5 & 9.4 & 1.86 & 11.8 & 17.5 & 12.5 & 1.6 & 2.4 & 2 & 21 & 20 & 8.2 & 6.5 & 0.79 \\
\hline TCH1065-E3 & 18.4 & 12.3 & 1.5 & 12.3 & 18.4 & 11.7 & 3.3 & 3.8 & 2.3 & 30 & 24 & 9.14 & 7.8 & 0.85 \\
\hline TCH1065-T25-L2 & 19.3 & 12.2 & 1.58 & 14 & 19.3 & 12.3 & 3 & 3 & 2.7 & 25 & 21 & 10.3 & 8 & 0.78 \\
\hline TCH1069-T1-R2 & 20 & 13 & 1.54 & 14 & 20 & 13.5 & 2.1 & 2.7 & 2.1 & 24 & 29 & 11.5 & 8.3 & 0.72 \\
\hline SCR1055-T2-L2 & 20 & 12 & 1.67 & 15 & 20 & 16 & 2.7 & 2.9 & 2.5 & 25 & 18 & 11.4 & 6 & 0.53 \\
\hline SCR1055-T3-L2 & 18 & 11 & 1.64 & 12 & 18 & 12 & 2.3 & 2.1 & 1.8 & 26 & 26 & 8.5 & 8.3 & 0.98 \\
\hline CHV1000-E4 & 16 & 8.5 & 1.88 & 11 & 16 & 10 & 1.8 & 2.3 & 1.7 & 21 & 22 & 8.1 & 6.1 & 0.75 \\
\hline CRO500-T10-L10 & 11 & 6.5 & 1.69 & 6 & 11 & 7 & 1.4 & 1.8 & 1.5 & 32 & 23 & 5.6 & 5.4 & 0.96 \\
\hline BEB500-T120-R5 & 17 & 15 & 1.13 & 13.5 & 17 & 14.5 & 3.5 & 3.2 & 2.5 & 30.4 & 34 & 13 & 5 & 0.38 \\
\hline BEB500-T120-R6 & 18 & 15.5 & 1.16 & 14.5 & 18 & 15 & 2.5 & 3.1 & 3 & 22 & 27 & 14.2 & 5.7 & 0.40 \\
\hline TCH1065-E124 & 19 & 15.5 & 1.23 & 13.5 & 19 & 15 & 3.3 & 4.5 & 3.5 & 27.5 & 26.5 & 14.4 & 7.5 & 0.52 \\
\hline TCH1065-E188 & 18 & 12.3 & 1.46 & 13.3 & 18 & 13 & 3.2 & 3.7 & 3.3 & 25 & 27 & 10 & 5.2 & 0.52 \\
\hline TCH1065-T21-R1 & 19.8 & 14.5 & 1.37 & 14.4 & 19.8 & 14.8 & 3.5 & 3.7 & 3.5 & 27 & 27 & 11.8 & 6.9 & 0.58 \\
\hline TCH1065-T15-R1 & 21.8 & 15 & 1.45 & 15.7 & 21.8 & 17.2 & 2.7 & 3.4 & 3.1 & 29 & 25 & 12 & 7.3 & 0.61 \\
\hline
\end{tabular}




\section{Table 2 (on next page)}

Variation in preservation grade and variation in maximum depth (difference between highest and lowest value) through the analysed trackways (see Table S1 for specific values of each track). 


\begin{tabular}{|c|c|c|c|c|c|c|c|c|c|}
\hline \multicolumn{5}{|c|}{ GRACILE MORPHOTYPE } & \multicolumn{5}{|c|}{ ROBUST MORPHOTYPE } \\
\hline Trackway & $\begin{array}{c}\text { Number } \\
\text { of } \\
\text { tracks }\end{array}$ & $\begin{array}{c}\text { Analysed } \\
\text { tracks }\end{array}$ & $\begin{array}{c}\text { Variation } \\
\text { in preservation } \\
\text { grade (min-max) }\end{array}$ & $\begin{array}{c}\text { Total } \\
\text { variation } \\
\text { in } \\
\text { depth }(\mathrm{mm})\end{array}$ & Trackway & $\begin{array}{l}\text { Number } \\
\text { of tracks }\end{array}$ & $\begin{array}{c}\text { Analysed } \\
\text { tracks }\end{array}$ & $\begin{array}{c}\text { Variation } \\
\text { in preservation } \\
\text { grade (min-max) }\end{array}$ & $\begin{array}{c}\text { Total } \\
\text { variation } \\
\text { in } \\
\operatorname{depth}(\mathrm{mm})\end{array}$ \\
\hline BEB500-T16 & 27 & 3 & $0.5-2.5$ (High) & 1.1 & BEB500-T75 & 71 & 2 & 0 & 0 \\
\hline BEB500-T17 & 120 & 4 & 1-2 (Medium) & 2.8 & $\begin{array}{l}\text { BEB500- } \\
\text { T120 }\end{array}$ & 29 & 4 & 0-2 (High) & 5.8 \\
\hline BEB500-T58 & 53 & 6 & 0.5-1.5 (Medium) & 2.3 & $\begin{array}{l}\text { TCH1065- } \\
\text { T15 }\end{array}$ & 2 & 2 & $0.5-2$ (High) & 1.5 \\
\hline BEB500-T73 & 18 & 4 & 1-2 (Medium) & 2 & TCH1069-T2 & 5 & 2 & 1-1.5 (Low) & 1.8 \\
\hline BEB500-T78 & 24 & 2 & 1-1 (Low) & 0.4 & & & & & \\
\hline BEB500-T82 & 59 & 2 & 1.5-1.5 (Low) & 1.9 & & & & & \\
\hline BEB500-T93 & 64 & 2 & 1-1.5 (Low) & 3.8 & & & & & \\
\hline CR0500-T10 & 75 & 14 & 0-2 (High) & 2.6 & & & & & \\
\hline $\begin{array}{c}\text { CRO500- } \\
\text { T30BIS }\end{array}$ & 11 & 5 & 0-2 (High) & 4.7 & & & & & \\
\hline $\mathrm{TCH} 1055-\mathrm{T} 2$ & 4 & 2 & 2-2.5 (Low) & 2.5 & & & & & \\
\hline TCH1065-T25 & 4 & 2 & 1-2 (Medium) & 2.7 & & & & & \\
\hline
\end{tabular}

